\title{
Error-landscape-based multiobjective calibration of the Smagorinsky eddy-viscosity using high-Reynolds-number decaying turbulence data
}

\author{
Johan Meyers, ${ }^{1, a)}$ Charles Meneveau, ${ }^{2, b)}$ and Bernard J. Geurts ${ }^{3, c)}$ \\ ${ }^{1}$ Department of Mechanical Engineering, Katholieke Universiteit Leuven, Celestijnenlaan 300A—Bus 2421, \\ B3001 Leuven, Belgium \\ ${ }^{2}$ Department of Mechanical Engineering, Johns Hopkins University, 3400 North Charles Street, \\ Baltimore, Maryland 21218, USA \\ ${ }^{3}$ Multiscale Modeling and Simulation, Mathematical Sciences, J. M. Burgers Center, University of Twente, \\ P.O. Box 217, 7500 AE Enschede, The Netherlands
}

(Received 14 June 2010; accepted 1 November 2010; published online 15 December 2010)

\begin{abstract}
A suite of large-eddy simulations (LESs) of decaying homogeneous isotropic turbulence at high Reynolds numbers is performed and compared to wind-tunnel experiments in the tradition of Comte-Bellot and Corrsin. The error-landscape approach is used for the evaluation of the Smagorinsky model, and the results are used to identify an optimal combination of model parameter and resolution in a statistically robust fashion. The use of experimental reference data in the error-landscape approach allows to evaluate the optimal Smagorinsky coefficient at high Reynolds numbers and to perform detailed comparisons with analytical predictions. We demonstrate, using a pseudospectral discretization, that the optimal so-called Smagorinsky trajectory obtained from the error-landscape analysis converges at high simulation resolutions to the high-Re theoretical Lilly prediction for the Smagorinsky coefficient. Using modified wavenumbers in the same spectral code, the current study also presents error-landscape results based on LES with "second-order" discretization errors. By slightly revising Lilly's analysis, we show that including the effect of numerical discretization when evaluating the strain-rate tensor needed in the subgrid-scale model leads to a good prediction of the optimal Smagorinsky parameter obtained from the corresponding error-landscape. Using similar analytical tools, we further demonstrate that the dynamic procedure can also be adapted to better account for the effects of discretization and test-filter shape. (C) 2010 American Institute of Physics. [doi:10.1063/1.3526758]
\end{abstract}

\section{INTRODUCTION}

In recent years, error behavior of large-eddy simulation (LES) and the assurance of quality in LES have gained considerable attention. ${ }^{1-3}$ These studies aim to formulate a rigorous standard for the assessment of accuracy and reliability in LES. Most of them use direct numerical simulations (DNSs) as point of reference since these provide very detailed information on flow properties. The use of DNS reference data for model development and evaluation has limited the applicability and understanding of error behavior in LES to relatively low-Reynolds numbers. In the current work, we apply the error-landscape approach ${ }^{3,4}$ in combination with high-Reynolds-number experimental reference data to extend our understanding of LES error behavior to a wider range of realistic flow conditions. Using this approach, we focus on the Smagorinsky model and investigate the effects of discretization errors on the optimal asymptotic value of the Smagorinsky coefficient.

The current work is based on the multiobjective error-

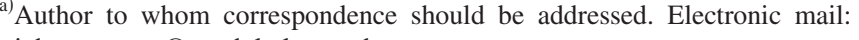
johan.meyers@mech.kuleuven.be.

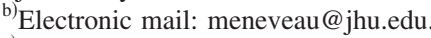

${ }^{c}$ Also at Anisotropic Turbulence, Fluid Dynamics Laboratory, Department of Applied Physics, Eindhoven University of Technology, P.O. Box 513, 5600 MB Eindhoven, The Netherlands. Electronic mail: b.j.geurts@utwente.nl.
}

landscape approach developed in the previous works. ${ }^{3,5}$ This approach provides a systematic framework to assess the errors of LES, characterizing the combined effects of modeling and discretization. In the current study, we use the decaying active-grid turbulence of Kang, Chester, and Meneveau, ${ }^{6}$ with initial Reynolds number $\operatorname{Re}_{\lambda}=716$, as reference. This provides a much higher Reynolds-number reference than the DNS reference employed in the previous studies. ${ }^{5}$ We perform the evaluation of LES quality using a series of flow properties that are sensitive to various scales of the flow. As in Ref. 3, this leads to a characterization of a multiobjective optimal refinement strategy. Second, for the current case, it is also shown that the error-landscape approach helps identify inconsistencies in boundary conditions at the large scales between the numerical setup and the experimental setup.

Since in prior studies the error-landscape approach was employed in combination with DNS reference data, ${ }^{3-5,7}$ lowReynolds-number effects were always playing a role in the eventual multiobjective optimal trajectories. ${ }^{3,4,8}$ This led, among others, to the formulation of a low-Reynolds-number correction to the Smagorinsky model. ${ }^{4,8}$ As will be shown in the current study, the optimal trajectories for the Smagorinsky model coefficient at high Reynolds number and high simulation resolution with spectral methods correspond quite well to Lilly's theoretical prediction for the Smagorinsky coefficient. ${ }^{8,9}$ Based on these observations, error-landscapes 
constructed with an alternate "second-order" discretization using modified wavenumbers in the same spectral code are also investigated in detail. For this case, we find that also in the presence of discretization errors, Lilly's analytical predictions can be employed when slightly adapted to appropriately include the effect of discretization.

The effect of discretization errors on large-eddy simulations has been a subject of study for many years., ${ }^{2,10-14}$ Lately, the focus is on situations where the filter width implied by the subgrid-scale models and the grid spacing do not differ greatly. ${ }^{2,3}$ In this case, computational resources are efficiently used, and the effects of numerical and modeling errors may be balanced as described in Refs. 5 and 12. The combined effect of model and discretization is often thought of as an implicit filter that smoothens the Navier-Stokes equations. This idea was originally formulated in the context of subgrid-scale-model effects only (without any discretization errors $)^{15-18}$ and also in the context of discretization effects only (without any model). ${ }^{19}$ However, it remains difficult to define such an implicit filter in a strict mathematical sense, which blocks progress in this direction-it is not even clear that such a "filter" would correspond to a well-defined operator. In order to simplify the analysis and avoid ambiguities, in this work we explicitly define the filter instead of presuming that an "implicit" filter is implied by model and discretization. We take a cubical sharp cut-off filter with width equal to the grid spacing. Although the shape of this filter does not explicitly enter the Smagorinsky subgrid-scale model, we consistently use it for a definition of filtered boundary conditions (in time and space) and for the definition of the filtered reference solution for the error-landscape approach. Using this filter, we employ Lilly's analysis for the determination of the Smagorinsky coefficient, presuming a $k^{-5 / 3}$ spectrum (multiplied with the selected LES filter shape). The effect of discretization then only enters as an additional filter function, as will be discussed in more detail in Sec. IV.

We believe that for high Reynolds number LES and high resolutions, any appropriate LES method (i.e., combination of filter, model, and discretization) should yield a $k^{-5 / 3}$ spectrum (multiplied with the selected/desired LES filter shape) along its multiobjective optimal refinement trajectory. In that case, the optimal Smagorinsky coefficient could correspond to the analytical prediction from Lilly's analysis. In our current study, this is observed for the combination of a cubical sharp cut-off filter, a Smagorinsky model, and either a spectral discretization or our "modified-wavenumber" discretization, which is of second-order accuracy. In these simulation conditions, the use of a dynamic Smagorinsky model instead of a standard Smagorinsky model should lead to a dynamic Smagorinsky coefficient, which also corresponds to Lilly's prediction. However, this is not always the case when the procedure is implemented straightforwardly. ${ }^{20}$ Therefore, presuming a $k^{-5 / 3}$ LES spectrum, we introduce correction factors in the terms of the dynamic procedure, which are analytically determined such that the procedure yields Lilly's prediction of the Smagorinsky coefficient. These correction factors take possible discretization errors in the Smagorinsky model and in the implementation of the dynamic procedure into account. A posteriori simulation results show that this leads to dynamic coefficients that much better match the multiobjective optimal refinement trajectory and Lilly's prediction.

This paper is further organized as follows. First, in Sec. II, the governing equation and case setup are discussed. Subsequently, the error-landscape methodology is discussed, and the results are presented for the pseudospectral and secondorder discretization in Sec. III. In Sec. IV, we present a discussion on Lilly's analysis (with some slight modifications for discretization effects), and the correspondence of analytical predictions with multiobjective optimal model coefficients (obtained in Sec. III) is addressed. Modifications to the dynamic procedure are also briefly elaborated. Finally, conclusions are presented in Sec. V.

\section{GOVERNING EQUATIONS AND CASE SETUP}

In this section, we present the governing equations and define the flow problem with which the LES error dynamics is analyzed.

\section{A. Governing equations}

Following the common LES approach, a low-pass convolution filter is first defined as

$$
\mathcal{G} \boldsymbol{u}(\boldsymbol{x})=\widetilde{\boldsymbol{u}}(\boldsymbol{x})=\iiint K_{\mathcal{G}}\left(\boldsymbol{x}-\boldsymbol{x}^{\prime}\right) \boldsymbol{u}\left(\boldsymbol{x}^{\prime}\right) \mathbf{d}^{3} \mathbf{x}^{\prime},
$$

with $K_{\mathcal{G}}$ being the filter kernel of the filter operator $\mathcal{G}$. Only normalized filters are considered such that $\mathcal{G} c=c$ for any constant function $c$. In Fourier space, the filter operation corresponds to $\widetilde{\boldsymbol{u}}(\boldsymbol{k})=G(\boldsymbol{k}) u(\boldsymbol{k})$, with $G(\boldsymbol{k})$ being the filter transfer function. In Fourier space, normalization of the filter requires $G(\mathbf{0})=1$.

The filtered Navier-Stokes equations for incompressible flows now follow as

$$
\begin{aligned}
& \nabla \cdot \tilde{\boldsymbol{u}}=0, \\
& \frac{\partial \widetilde{\boldsymbol{u}}}{\partial t}+\nabla \cdot(\tilde{\boldsymbol{u}} \otimes \tilde{\boldsymbol{u}})+\nabla \tilde{p}-\frac{1}{\operatorname{Re}} \nabla^{2} \tilde{\boldsymbol{u}}+\nabla \cdot \boldsymbol{\tau}=0,
\end{aligned}
$$

with $\tilde{\boldsymbol{u}}$ being the filtered velocity field, $\tilde{p}$ the filtered pressure field, and Re the Reynolds number. Moreover, $\boldsymbol{\tau}$ is the subgrid-scale stress tensor,

$$
\boldsymbol{\tau}=\widetilde{\boldsymbol{u} \otimes \boldsymbol{u}}-\tilde{\boldsymbol{u}} \otimes \tilde{\boldsymbol{u}},
$$

which is unclosed in terms of $\tilde{\boldsymbol{u}}$.

In the current study, we focus on the Smagorinsky model, ${ }^{21}$ which represents the subgrid-scale stresses as

$$
\boldsymbol{\tau}-\frac{1}{3} \operatorname{tr}(\boldsymbol{\tau}) \boldsymbol{I} \rightarrow \boldsymbol{m}=-2\left(C_{S} \Delta\right)^{2}\langle 2 \boldsymbol{S}: \boldsymbol{S}\rangle^{1 / 2} \boldsymbol{S},
$$

with $\boldsymbol{S}=\left[\nabla \tilde{\boldsymbol{u}}+(\nabla \tilde{\boldsymbol{u}})^{T}\right] / 2$ being the filtered rate-of-strain tensor. ${ }^{40}$ The operator $\langle\cdot\rangle$ represents the ensemble averaging operator. For homogeneous turbulence, it corresponds to spatial averaging over a box in the limit of its size going to infinity. 


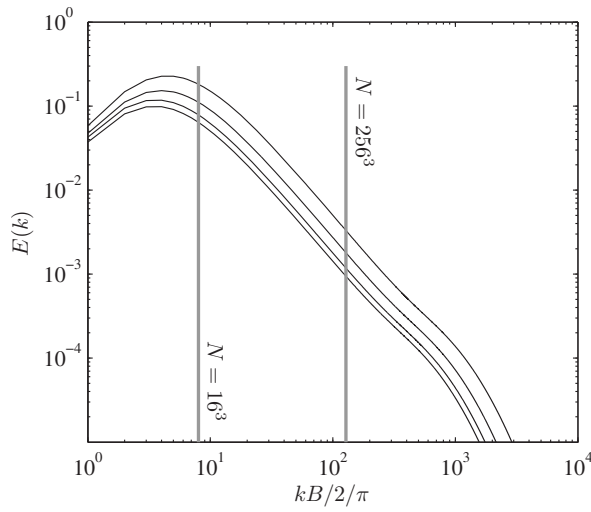

FIG. 1. Energy spectra fitted to the experiment of Kang et al. (Ref. 6) at four different measurement stations (from top to bottom, the spectra correspond to measurement stations $x / M=20,30,40,48)$. The cut-off wavenumbers related to smallest $\left(16^{3}\right)$ and largest $\left(256^{3}\right)$ resolution in the LES database are marked in gray.

In our simulations, we approximate it by spatial averaging over the simulation domain. The $\operatorname{trace} \operatorname{tr}(\boldsymbol{\tau})$ is not modeled directly, but absorbed in the pressure, as is common practice in incompressible LES.

The formulation of the Smagorinsky model in Eq. (5) using $\langle 2 \boldsymbol{S}: \boldsymbol{S}\rangle^{1 / 2}$ differs slightly from what is most often used, namely, a local approach using $(2 S: S)^{1 / 2}$. The mean eddyviscosity approach is more straightforward to implement in a spectral method (as we are using) and further has the advantage that it leads to exact expressions when Lilly's analysis of the Smagorinsky model is considered (the disadvantage is that the model is less general for complex geometry applications). When the results in the current paper are compared to a classical Smagorinsky implementation and, in particular, when the levels of Smagorinsky coefficients are compared, the difference in average dissipation between both formulations should be taken into account. This amounts to a correction of the Smagorinsky coefficients by a factor $\left(\langle 2 S: S\rangle^{3 / 2} /\left\langle(2 S: S)^{3 / 2}\right\rangle\right)^{1 / 2}$. The ratio is close to 1: e.g., McMillan and Ferziger ${ }^{22}$ were among the first to analyze this. A more precise determination of this ratio was given by Cerutti et al.: ${ }^{23}$ they report $\left\langle(2 S: S)^{3 / 2}\right\rangle /\langle 2 S: S\rangle^{3 / 2} \approx 1.2$ (the coefficient $\beta$ shown as squares in their Fig. 14), leading to a factor $1 /(1.2)^{1 / 2}$ between Smagorinsky coefficients of the two formulations. Hence, much of the information found in the remainder of this study on the value of the Smagorinsky coefficient may be corrected by this factor when applied to a Smagorinsky implementation based on $(2 \boldsymbol{S}: S)^{1 / 2}$.

\section{B. Setup of the LES database}

For our reference data, we rely on the active grid experiment of Kang et al., ${ }^{6}$ which provides spectra of decaying turbulence at four different measurement stations in a wind tunnel $(x / M=20,30,40,48$, where $M=15.2 \mathrm{~cm}$ is the mesh size of the grid). For the large-eddy simulations in the current work, experiments are translated into a time frame using the Taylor hypothesis and representing the flow in a frame convecting downstream at the mean velocity. The first measurement station $\left(\operatorname{Re}_{\lambda}=716\right)$ is used to generate the initial condition; data from the next measurement stations $\left(\operatorname{Re}_{\lambda}=676,650,626\right)$ are used as reference for the assessment of the numerical solution. Simulations are performed in a box with size $B=10 L$, with $L \equiv E^{3 / 2} / \varepsilon$ being the integral length scale evaluated at the initial measurement station (this yields $L \approx 3.5 M$ ). For the setup of the LES, we follow the procedure set out in Ref. 6, with the difference that the experimentally obtained energy spectra are fitted with new functional forms that provide better representations of the spectra. ${ }^{24}$ The fitted spectra at the four different Reynolds numbers are displayed in Fig. 1.

Large-eddy simulations are performed using a Smagorinsky model [Eq. (5)]. Two spatial discretization schemes are compared: (1) a classical pseudospectral method ${ }^{25}$ and (2) an alternate discretization, which is constructed in the same spectral code using wavenumber modifications $s^{10,26}$ and is of second-order accuracy. In the latter case, the first and second derivatives of a Fourier mode $f=\exp (\boldsymbol{l k x})$, with $l^{2}=-1$, are expressed as

$$
\begin{aligned}
& \frac{\partial f}{\partial x_{i}} \approx l \frac{\sin \left(k_{i} \Delta / 2\right)}{\Delta / 2} \exp (\iota \boldsymbol{k} \boldsymbol{x}), \\
& \frac{\partial^{2} f}{\partial x_{i}^{2}} \approx \frac{2 \cos \left(k_{i} \Delta\right)-2}{\Delta^{2}} \exp (\iota \boldsymbol{k} \boldsymbol{x})
\end{aligned}
$$

instead of using $\imath k_{i} \exp (\imath \boldsymbol{k} \boldsymbol{x})$ and $-k_{i}^{2} \exp (\imath \boldsymbol{k} \boldsymbol{x})$ normally employed in the spectral codes. The definition of the first and second derivatives in Eq. (6) is such that they introduce a finite-difference-like second-order error in the formulation of the subgrid and viscous terms of our equations. This is important for further analysis in Sec. IV of the current work. For the convective terms and pressure terms, we also use the first derivatives as expressed in Eq. (6) such that we have the same order of accuracy for these terms. This particular choice ensures a stable projection operator in our spectral code but is not representative for normal second-order finitedifference or finite-volume discretizations, where additional interpolation schemes play an important role in the discretization of the convective terms. Equations are integrated in time with a four-stage fourth-order Runge-Kutta scheme.

For the initialization of the LES field and for the definition of the filtered experimental reference, a cubical sharp cut-off filter is used, which corresponds to the cutoff of the pseudospectral discretization. Consequently, the filter width $\Delta=\pi / k_{c}$ (with $k_{c}$ being the grid cutoff) used in the Smagorinsky model corresponds to $B / N$, with $B$ being the size of the computational box $(B=10 L)$ and $N^{3}$ the grid size. To setup the LES database, a systematic variation of the grid size $N^{3}\left(16^{3}-256^{3}\right)$ and the model coefficient $C_{s}(0-0.284)$ is performed such that the error behavior can be properly charted as a function of these parameters. In total, 14 different simulation resolutions and 31 different coefficients are included such that a comprehensive representation of the error landscape is obtained. 


\section{ERROR-LANDSCAPES}

The error-landscape methodology is briefly reviewed in Sec. III A. Subsequently, error-landscape results and multiobjective optimal refinement trajectories are presented both for the pseudospectral discretization and the second-order discretization in Sec. III B.

\section{A. The error-landscape approach}

The error-landscape approach was introduced in Refs. $3-5$ as a tool for a comprehensive numerical evaluation of LES error behavior relying on a large database of large-eddy simulations and an experimental or numerical reference. Central to this approach is the use of a range of error measures characterizing different scales in the solution. ${ }^{3,4}$ For the evaluation of error behavior in decaying homogeneous isotropic turbulence, two different sets of error definitions were employed, i.e.,

$$
\begin{aligned}
& D_{p}\left(N, C_{s}\right)=\left[\frac{\int_{0}^{T}\left\{\int_{0}^{k_{c}} k^{p}\left[E_{\mathrm{LES}}(k, t)-E_{\mathrm{ref}}(k, t)\right] \mathrm{d} k\right\}^{2} \mathrm{~d} t}{\int_{0}^{T}\left[\int_{0}^{k_{c}} k^{p} E_{\mathrm{ref}}(k, t) \mathrm{d} k\right]^{2} \mathrm{~d} t}\right]^{1 / 2}, \\
& d_{p}\left(N, C_{s}\right)=\left\{\frac{\int_{0}^{T} \int_{0}^{k_{c}} k^{2 p}\left[E_{\mathrm{LES}}(k, t)-E_{\mathrm{ref}}(k, t)\right]^{2} \mathrm{~d} k \mathrm{~d} t}{\int_{0}^{T} \int_{0}^{k_{c}} k^{2 p}\left[E_{\mathrm{ref}}(k, t)\right]^{2} \mathrm{~d} k \mathrm{~d} t}\right\}^{1 / 2},
\end{aligned}
$$

with $k_{c}=\pi / \Delta$ being the grid cut-off frequency. Both $D_{p}$ and $d_{p}$ provide an error measure, which is based on weighted integrals of the LES energy spectrum compared to the reference spectrum and properly normalized. In wavenumber space, the upper integration bound corresponds to the grid cutoff $k_{c}$ such that the LES results are compared to the reference results up to the LES grid cutoff. This is equivalent to comparing the LES data to the sharp-cut-off-filtered reference data. Depending on the values of $p$, the approach defines errors on large-scale properties $(p=-1,0)$ or on resolved fine-scale properties $(p=1,2)$. Remark that Eqs. (7) and (8) are defined using a spherical sharp cut-off filter instead of the cubical sharp cut-off filter used in the simulations. This has the advantage of a practical and elegant error definition, while the impact on the results in Sec. III B is small, as is elaborated in more detail at the end of the current section.

Of the two definitions in Eqs. (7) and (8), $D_{p}$ is the one that is most easily linked to physical flow properties: for $p=-1,0$, and 2 , it is readily shown that $D_{p}$ represents the relative error on the integral length scale $\mathcal{L}$, the resolved turbulent kinetic energy $E$, and the resolved enstrophy $\mathcal{E}$ (cf. Ref. 3 for details), respectively. The other error definition $d_{p}$ is a more robust error measure: as is appreciated form the definition in Eq. (7), $D_{p}$ is based on a quadratic weighting of errors but does not incorporate a penalization for incorrect distributions of underlying energy spectra in wavenumber space. In Ref. 3, this was shown to lead to incorrect characterization of LES quality when only a few errors are included in the analysis. The definition of $d_{p}$ [Eq. (8)] remedies this effect as it defines a mathematical norm in which errors at each wavenumber contribute positively to the total.

Based on either $D_{p}\left(N, C_{s}\right)$ or $d_{p}\left(N, C_{s}\right)$, an optimal refinement trajectory $\hat{C}_{s}^{(p)}(N)$ is defined as the value $C_{s}$, which provides a minimal error $D_{p}$ (or $d_{p}$ ) at given resolution $N$. Obviously, $\hat{C}_{s}^{(p)}(N)$ may depend on $p$, and hence, a multiobjective approach, where errors at different $p$ are considered simultaneously, is called for. To this end, near-optimal regions related to $D_{p}\left(N, C_{s}\right)$ are defined as ${ }^{3}$

$$
\Omega_{p}(a)=\left\{\begin{array}{l|l}
N \in \mathbb{N} ; C_{s} \in \mathbb{R}^{+} \mid \frac{D_{p}\left(N, C_{s}\right)}{D_{p}\left(N, \hat{C}_{s}^{(p)}(N)\right)} \leq a
\end{array}\right\},
$$

where we select $^{3} \quad a=1.2$. Further, we recall that a global weighted error is defined as

$$
\tilde{D}\left(N, C_{s}\right)=\frac{\sum_{p}\left\{D_{p}\left(N, C_{s}\right) / D_{p}\left(N, \hat{C}_{s}^{(p)}(N)\right)\right\}}{\sum_{p}\left\{1 / D_{p}\left(N, \hat{C}_{s}^{(p)}(N)\right)\right\}},
$$

Based on $\widetilde{D}\left(N, C_{s}\right)$, a multiobjective optimal refinement trajectory $\widetilde{C}_{s}(N)$ is defined and similar definitions are used for $d_{p}$. In Ref. 3 , it was shown that both error definitions $D_{p}$ and $d_{p}$ lead to roughly the same multiobjective optimal refinement trajectory provided a sufficient number of errors (at different $p$ ) are included in Eq. (10). The results shown in Sec. III B confirm this observation.

In the current study, the reference data correspond to high Reynolds number wind-tunnel data instead of direct numerical simulations. A practical consequence is that less detailed data is available; in particular, energy spectra are only given at four different measurement locations, of which one is used for LES initialization. Therefore, the time integrals in Eqs. (7) and (8) cannot be used. Instead, we chose to replace them by a sum over the three measurement locations available for comparison. Using Taylors hypothesis and $t_{i}=x_{i} / U$, with $U$ being the mean streamwise wind-tunnel velocity and $x_{i}(i=1,2,3)$ corresponding to the measurement locations used for evaluation $(x / M=30,40,48)$, this leads to

$$
\begin{aligned}
& D_{p}\left(N, C_{s}\right)=\left[\frac{\sum_{t_{i}}\left\{\int_{0}^{k_{c}} k^{p}\left[E_{\mathrm{LES}}\left(k, t_{i}\right)-E_{\mathrm{expt}}\left(k, t_{i}\right)\right] \mathrm{d} k\right\}^{2}}{\sum_{t_{i}}\left[\int_{0}^{k_{c}} k^{p} E_{\text {expt }}\left(k, t_{i}\right) \mathrm{d} k\right]^{2}}\right]^{1 / 2}, \\
& d_{p}\left(N, C_{s}\right)=\left[\frac{\sum_{t_{i}} k_{0}^{k} k^{2 p}\left(E_{\mathrm{LES}}\left(k, t_{i}\right)-E_{\mathrm{EXP}}\left(k, t_{i}\right)\right)^{2} \mathrm{~d} k}{\sum_{t_{i}} \int_{0}^{k_{c}} k^{2 p}\left(E_{\mathrm{EXP}}\left(k, t_{i}\right)\right)^{2} \mathrm{~d} k}\right]^{1 / 2} .
\end{aligned}
$$

Obviously, error measures are now based on considerably less data than the evaluation of Eqs. (7) and (8) when DNS reference data are available for a large number of instances in time. In Sec. III B, Eqs. (11) and (12) are used for the elaboration of near-optimal regions and multiobjective optimal refinement trajectories.

As already mentioned above, the definitions of the errors in Eqs. (7) and (8) compare LES field and reference field both filtered with a spherical sharp cut-off filter instead of the cubical sharp cut-off filter used in the simulations (where the definition of the initial field and the dealiasing operator use a 

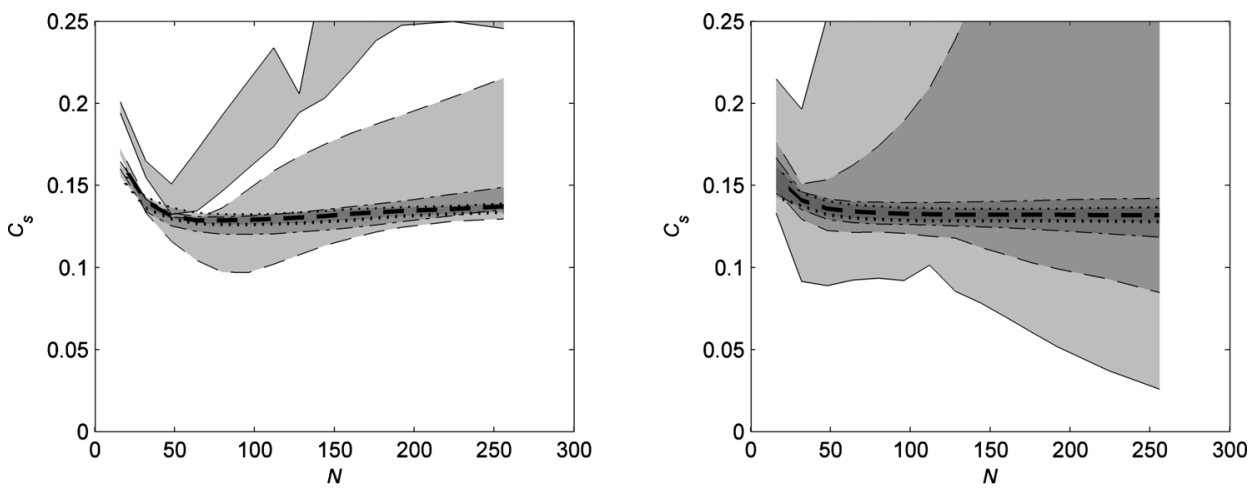

(a)

(b)

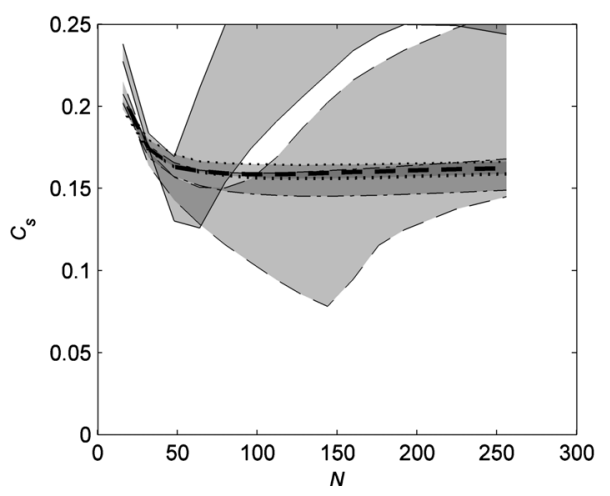

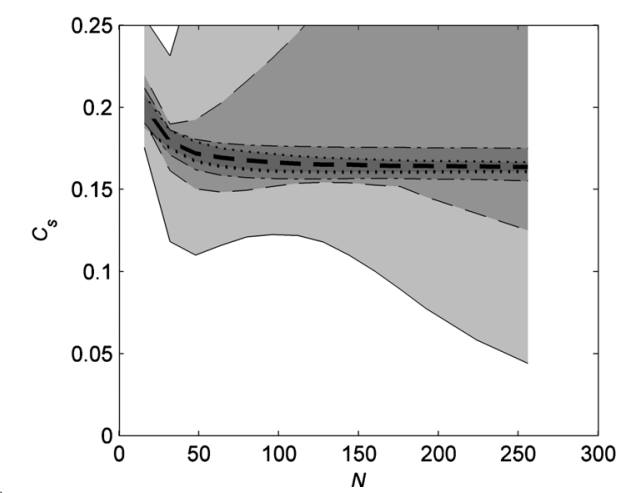

(d)

FIG. 2. "Near-optimal" regions of Smagorinsky LES in the experiment of Kang et al. shown using shaded gray but also semitransparent such that areas with overlapping near-optimal regions appear with darker shades of gray. The curves,,$----\cdot$, and $\cdots$, respectively, mark the boundaries of the $\Omega_{p}(p=-1$ to 2$)$ : [top: (a) and (b)] spectral discretization, [bottom: (c) and (d)] second-order discretization, [left: (a)-(c)] based on errors $D_{p}$ [Eq. (11)], [right: (b)-(d)] based on errors $d_{p}$ [Eq. (12)], and (bold dashed line) multiobjective refinement strategies.

cubical sharp cut-off filter). Hence, scales located in the corners of the cube in Fourier space that fall outside the sphere with radius $k_{c}$ are not included in the postprocessing of errors. For the definition of multiobjective optimal refinement trajectories, this difference in definition has negligible impact on the results. By using a more involved three-dimensional error definition including the corners, we have found that the difference in the $\widetilde{C}_{s}(N)$ trajectories is largest at low resolutions but remains below 5\% (and below 3\% for $N \geq 128$ ). This complies with the idea that the characterization of multiobjective optimal refinement trajectories is robust to the specific details of error definition provided a sufficient number of errors, related to different scales in the flow, are included in the weighted error. We prefer the definitions provided in Eqs. (7) and (8) for their ease of use compared to a more complicated error definition that formally includes the extra corner modes in Fourier space.

\section{B. Error-landscape results and multiobjective strategies}

Error-landscape results are presented based on a large set of LES at different $\left(N, C_{s}\right)$ combinations. Near-optimal regions are discussed for the pseudospectral and for our second-order discretization. Multiobjective refinement trajectories are compared.

First, in Fig. 2, near-optimal regions are presented. The figure has four parts. Parts (a) and (b) show the near-optimal regions for $p=-1,0,1$, and 2 obtained from pseudospectral
LES, respectively, using $D_{p}$ [Eq. (11)] and $d_{p}$ [Eq. (12)] as error definition. Similarly, near-optimal regions obtained from our second-order LES runs are shown in parts (c) and (d). We first focus on the results of the pseudospectral discretization [Figs. 2(a) and 2(b)]. We see that near-optimal regions for $D_{0}, D_{1}$, and $D_{2}$ overlap; similarly, all nearoptimal regions based on $d_{p}$ overlap. Both multiobjective optimal refinement trajectories $\widetilde{C}_{s}(N)$ are located inside these multiobjective overlap regions. However, it is striking that the near-optimal region for $D_{-1}$ (representing an error that is predominantly based on the large scales of the solution) has no significant intersection with the other near-optimal regions. We believe that this points toward an inconsistency between the experimental and the numerical setups. In the current case, the effect of the sidewalls in the wind tunnel on the largest flow scales are not properly represented in the numerical simulation, where periodic boundary conditions are employed. Since the box size $B$ in our simulations is considerably larger than the integral length scale $L(B / L$ $\approx 10$ ), this effect is seen to not strongly influence the smaller-scale predictions (emphasized by $p=0-2$ ). When looking at the results of the second-order discretization, the same trends are observed: all near-optimal regions overlap, except for $D_{-1}$. Using Eq. (10), multiobjective optimal refinement trajectories are readily defined, and these respective trajectories are also displayed in Fig. 2.

To further assess the quality of results, we investigate some spectra along multiobjective optimal refinement trajec- 

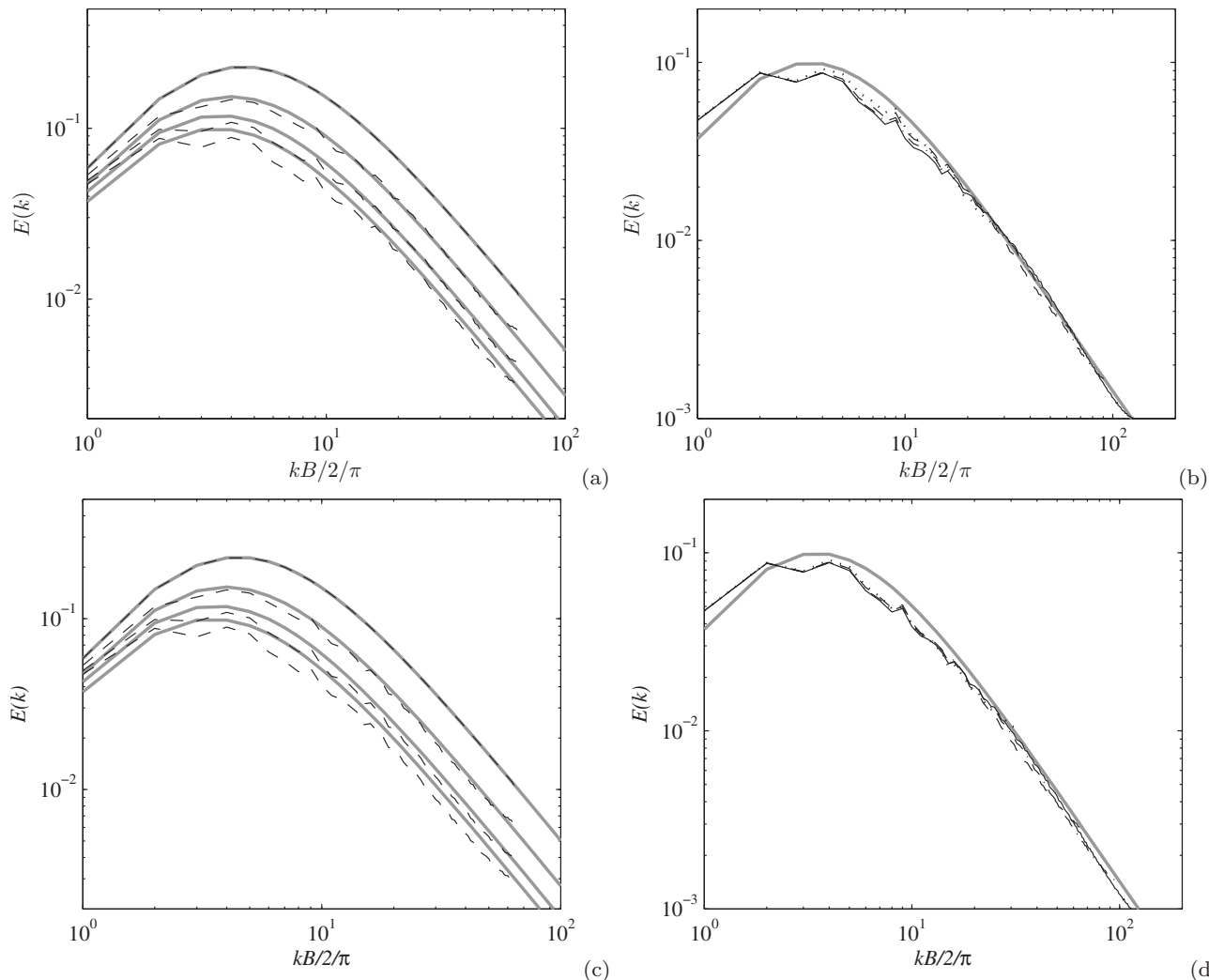

FIG. 3. Comparison of experiments (bold gray lines) with LES spectra at multiobjective optimal $C_{s}$ values using spectral discretization [(a) and (b)] and second-order discretization [(c) and (d)]. [(a) and (c)] LES with a resolution of $128^{3}$ (dashed lines) compared to the experimental data at different measurement stations $(x / M=20,30,40,48)$. [(b) and (d)] LES with respective resolutions of $64^{3}(\cdots), 128^{3}(--), 192^{3}(-\cdot)$, and $256^{3}(-)$ at the final measurement station $(x / M=48)$.

tories in Fig. 3. First of all, in Figs. 3(a) and 3(b), it is appreciated that the results of the pseudospectral discretization lead to a well-defined $k^{-5 / 3}$ range at all measurement stations and resolutions shown. These inertial-range spectra further correspond well to the experimental spectra, which are also displayed. The large-scale part of the spectrum (at low wavenumber) is not predicted properly for any of the simulations. We believe that this is related to inconsistencies in boundary conditions between experiments and simulations (as discussed above). Overall, the results for the rest of the spectrum are quite satisfactory for simulations near $\widetilde{C}_{s}(N)$. For the second-order results in Figs. 3(c) and 3(d), the same trends are observed. A well-defined $k^{-5 / 3}$ range is found in the spectra, and the match to the experimental reference data is satisfactory, but slightly worse compared to the pseudospectral results.

In Fig. 4, the multiobjective optimal refinement trajectories are plotted for both discretizations and both error measures. It is appreciated that $\widetilde{C}_{s}$ either evaluated using $D_{p}$ or $d_{p}$ roughly leads to the same result. At low resolutions, the optimal coefficient depends on the simulation resolution. In our opinion, this is related to the marginal resolution of the integral length scale $L / \Delta<10$. For $N>100$ and $L / \Delta>10, \widetilde{C}$ appears to have a horizontal asymptote. Using the $d_{p}$-error definition, this asymptotic value roughly corresponds to $C_{s}=0.132$ for the pseudospectral results. This also corresponds well to the Smagorinsky coefficient, which can be obtained by using Lilly's analysis with a cubical sharp cutoff filter, ${ }^{8}$ for which we find $C_{s}=0.135$. For the second-order results (also using $d_{p}$ ), we find an asymptotic value of $C_{s}=0.164$, which is considerably higher than the pseudospectral value. Also, this value can be obtained analytically by including discretization effects into Lilly's analysis.

This is further discussed in Sec. IV.

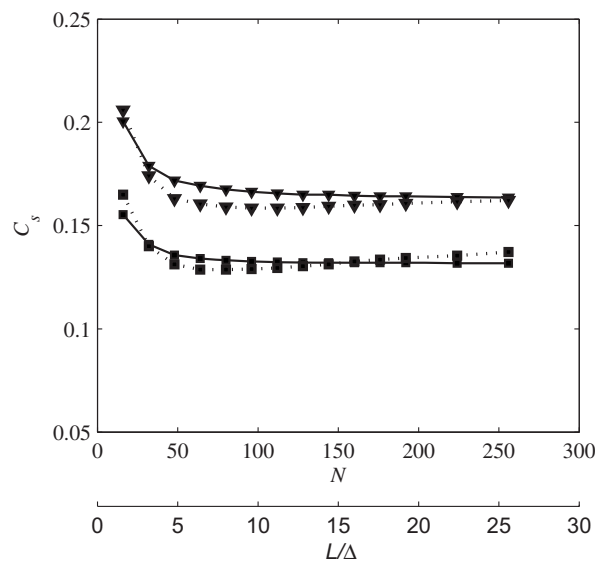

FIG. 4. Multiobjective optimal trajectories for the pseudospectral method $(\boldsymbol{\square})$ and the second-order method $(\boldsymbol{\nabla})$ : $\left(()\right.$ using $d_{p} ;(\cdots)$ using $D_{p}$. 


\section{ANALYSIS OF THE ASYMPTOTIC OPTIMAL SMAGORINSKY COEFFICIENT}

We discuss the asymptotic values obtained from the multiobjective optimal trajectories at high simulation resolutions by means of Lilly's analysis. First, in Sec. IV A, Lilly's analysis is briefly reviewed and slightly revised to include the effect of discretization errors. The correspondence of analytical results and the "measured" optimal Smagorinsky coefficient is discussed. Second, in Sec. IV B, the same type of approach is used to elaborate the effect of discretization in the dynamic Smagorinsky model.

\section{A. The Smagorinsky model}

From a theoretical point of view, many statistical constraints may be imposed on a subgrid-scale model ${ }^{27}$ However, for a basic model such as the Smagorinsky model, in the possible presence of discretization errors, probably the only statistical constraint that may be realized in practice is the requirement that the model sheds the correct amount of energy in time. Hence, at large Reynolds numbers,

$$
\left(C_{s} \Delta\right)^{2}\langle 2 S: S\rangle^{3 / 2}=\langle-\boldsymbol{m}: S\rangle=\varepsilon,
$$

with $\varepsilon$ being the total turbulent dissipation (for $\operatorname{Re} \gg 1$, we presume that the effect of viscosity can be neglected in the LES energy balance).

Lilly ${ }^{9}$ used this idea in his analysis of the Smagorinsky model and its constant. By presuming an energy spectrum $E(k)=C_{K} \varepsilon^{2 / 3} k^{-5 / 3}$ (with $C_{K}$ being the Kolmogorov constant) and expressing for homogeneous isotropic turbulence (cf. Appendix A for details)

$$
\langle\boldsymbol{S}: \boldsymbol{S}\rangle=\frac{1}{4 \pi} \iiint_{-\infty}^{\infty} C_{K} \varepsilon^{2 / 3} k^{-5 / 3}[G(\boldsymbol{k})]^{2} \mathrm{~d} \boldsymbol{k}
$$

[with $G(\boldsymbol{k})$ being the transfer function of the LES low-pass filter], the dissipation $\varepsilon$ can be canceled from Eqs. (13) and (14). This yields ${ }^{8}$

$$
C_{s} \Delta=\frac{C_{s, \infty} \Delta}{\gamma},
$$

with

$$
C_{s, \infty}=\frac{1}{\pi}\left(\frac{2}{3 C_{K}}\right)^{3 / 4}
$$

and

$$
\gamma=\frac{\left(\frac{1}{3 \pi} \iiint_{-\infty}^{\infty} k^{-5 / 3}[G(\boldsymbol{k})]^{2} \mathrm{~d} \boldsymbol{k}\right)^{3 / 4}}{\pi / \Delta} .
$$

In Lilly's original analysis, ${ }^{9}$ the LES low-pass filter is a spherical sharp cut-off filter for which $\gamma=1$. Taking a value of $C_{K}=1.6$ for the Kolmogorov constant then leads to $C_{s}=C_{s, \infty}=0.165$. For other filter shapes, $\gamma$ needs to be determined by numerical integration (cf. Ref. 8) and $C_{s}=0.165 / \gamma$. Elaboration for a cubical sharp cut-off filter ${ }^{8}$ leads to $\gamma=1.22$ and $C_{s}=0.135$. This value corresponds very well to the asymptotic $C_{s}$ value of the multiobjective optimal refinement trajectory at high simulation resolutions in Fig. 2 (as discussed in Sec. III B).

To express the model dissipation in Eqs. (13) and (14), Lilly's analysis relies on a $k^{-5 / 3}$ spectrum, which is the expected spectral behavior at high-Reynolds-number LES. Consequently, it is no surprise that the multiobjective optimal Smagorinsky coefficient of the pseudospectral method at high resolution corresponds well to this theoretical prediction. It merely reflects that the Smagorinsky model performs reasonably well for this test case, Reynolds number, and simulation resolution, yielding a $k^{-5 / 3}$ spectrum that closely matches the experimental data (as clear from Fig. 3 and also demonstrated by many other authors, see, e.g., Refs. 6, 17, and 18).

Also for Smagorinsky LES with second-order discretization, reasonable LES results may be obtained but with a different location of the multiobjective optimal refinement trajectory (see Fig. 4 and Ref. 3). If we presume that secondorder Smagorinsky LES is sufficiently accurate to also yield spectra that match high-Reynolds-number experimental data, then Lilly's analysis can be straightforwardly extended. The main difference is that we have to take discretization errors into account, but acting on a "known" $k^{-5 / 3}$ spectrum. Hence, the only discretization effect entering into the analysis is the discrete representation of $\langle\boldsymbol{S}: \boldsymbol{S}\rangle$ and $\boldsymbol{S}$ (denoted here by $\boldsymbol{S}_{\boldsymbol{d}}$ ), i.e., in this case, possible discretization errors on the convective terms do not impact on the dissipation balance used in Lilly's analysis. The effect of discretization errors on the strain-rate tensor is readily incorporated into Eqs. (14) and (17) by means of an additional filter, ${ }^{28}$ which follows from a spectral analysis of $\left\langle S_{d}: S_{d}\right\rangle$. Further elaboration of $\left\langle S_{d}: S_{d}\right\rangle$, leading to an expression that is similar to Eq. (14), is presented in Appendix A [Eq. (A11)]. Based on this expression for $\left\langle S_{d}: S_{d}\right\rangle$ [Eq. (A11)], Eq. (17) is then replaced with

$$
\gamma_{d}=\frac{\left(\frac{1}{3 \pi} \iiint_{-\infty}^{\infty} k^{-5 / 3}[G(\boldsymbol{k})]^{2} G_{D}(\boldsymbol{k}) \mathrm{d} \boldsymbol{k}\right)^{3 / 4}}{\pi / \Delta},
$$

with $G_{D}(\boldsymbol{k})$ being the filter induced by the discretization of $\langle\boldsymbol{S}: \boldsymbol{S}\rangle$. Using the modified wavenumbers for a secondorder discretization [Eq. (6)], $G_{D}(\boldsymbol{k})$ corresponds to (cf. Appendix A)

$$
G_{D}(\boldsymbol{k})=2 \frac{\sum_{i=1}^{3} 1-\cos \left(k_{i} \Delta\right)}{(k \Delta)^{2}} .
$$

Integration of Eq. (18) using this filter leads to $\gamma_{d}=1.005$, and hence, $C_{s}=C_{s, \infty} / \gamma_{d} \approx 0.165$. This value corresponds very well to the multiobjective optimal asymptotic Smagorinsky constant observed in Fig. 4 for high simulation resolutions and the second-order discretization. Moreover, in Fig. 3, it is shown for our second-order results that the spectra at multiobjective $C_{s}$ values display a well-defined $k^{-5 / 3}$ range, justifying the presumptions made to extend Lilly's analysis above. From this analysis, it is clear that the optimal Smagorinsky coefficient in the presence of second-order discretization effects is a factor $\gamma / \gamma_{d}=1.21$ higher than the "pseudospectral" coefficient. 
The above result re-establishes Lilly's analysis as a tool for the calibration of model coefficients even in the presence of discretization errors. This presumes that the multiobjective optimal trajectory of the LES corresponds well to a $k^{-5 / 3}$ spectrum at high simulation resolutions and Reynolds numbers. We believe that this is a minimum requirement for the quality and reliability of a LES method and, consequently, that the asymptotic convergence of the LES's multiobjective optimal trajectory (for $\operatorname{Re} \gg 1$ and $N \gg 1$ ) to the "Lilly value" is a useful verification element for any LES method.

\section{B. The dynamic Smagorinsky model}

Similar to the Smagorinsky model, discretization errors may affect the dynamic procedure, leading to incorrect predictions of the optimal trajectories. ${ }^{20}$ Based on the results in Sec. IV A, the dynamic procedure as introduced by Germano et $a l .{ }^{29}$ is now corrected. As before, we presume that the LES method is sufficiently reliable to yield a $k^{-5 / 3}$ spectrum when the correct model coefficient is selected by the dynamic procedure. Two issues are addressed: first of all, the effect of discretization errors and, second, the effect of a test filter that is not scale-similar to the LES filter (as may be often the case in discrete implementations of the test filter).

First, we start with briefly reviewing some elements of the dynamic procedure. Germano's dynamic procedure requires the use of a test filter $\mathcal{H}$ which is applied on the resolved flow field $\tilde{\boldsymbol{u}}$ and is defined here as

$$
\mathcal{H} \widetilde{\boldsymbol{u}}(\boldsymbol{x})=\overline{\tilde{\boldsymbol{u}}}(\boldsymbol{x})=\int K_{H}\left(\boldsymbol{x}-\boldsymbol{x}^{\prime}\right) \widetilde{\boldsymbol{u}}\left(\boldsymbol{x}^{\prime}\right) \mathbf{d}^{3} \mathbf{x}^{\prime},
$$

with $K_{H}$ being the filter kernel of $\mathcal{H}$. In Fourier space, Eq. (20) corresponds to

$$
\overline{\tilde{\boldsymbol{u}}}(\boldsymbol{k})=H(\boldsymbol{k}) \widetilde{\boldsymbol{u}}(\boldsymbol{k})=H(\boldsymbol{k}) G(\boldsymbol{k}) \boldsymbol{u}(\boldsymbol{k}) .
$$

The filter $\mathcal{H}$ is considered scale-similar to $\mathcal{G}$ if

$$
H(\boldsymbol{k}) G(\boldsymbol{k})=G(n \boldsymbol{k}),
$$

with $n>0$ being the ratio between the widths of the $\mathcal{H} * \mathcal{G}$ filter and $\mathcal{G}$ the filter. For practical use in the context of the dynamic procedure, $n=2$ is commonly selected. More on scale-similarity of test filters is, e.g., found in Ref. 30.

The dynamic procedure employs Germano's identity, ${ }^{31}$ which expresses a relation between the subgrid-scale stress tensor $\mathbf{T}$ of the $(\mathcal{H} * \mathcal{G})$-filtered Navier-Stokes equations and the stress tensor $\tau$ [cf. Eq. (4)], i.e.,

$$
L \equiv \boldsymbol{T}-\overline{\boldsymbol{\tau}}=\overline{\tilde{\boldsymbol{u}} \otimes \tilde{\boldsymbol{u}}}-\overline{\tilde{\boldsymbol{u}}} \otimes \overline{\tilde{\boldsymbol{u}}} .
$$

The right-hand side of this equation is fully resolved on the $\mathcal{G}$-filter level and can be evaluated during simulations. Hence, by inserting a Smagorinsky model $\mathbf{M}$ as model for $\mathbf{T}$ and $\mathbf{m}$ for $\boldsymbol{\tau}$ and by presuming that both models operate with the same model constant $C_{\mathrm{dyn}}$, one obtains a tensorial equality, which may be used to estimate $C_{\mathrm{dyn}}$,

$$
\mathbf{L} \approx \mathbf{M}-\overline{\mathbf{m}}=C_{\mathrm{dyn}} \Delta^{2} \mathbf{Q},
$$

with

$$
\boldsymbol{Q}=-\left[2 n^{2}\langle 2 \overline{\boldsymbol{S}}: \overline{\boldsymbol{S}}\rangle^{1 / 2} \overline{\boldsymbol{S}}-2 \overline{\langle 2 \boldsymbol{S}: \boldsymbol{S}\rangle^{1 / 2} \boldsymbol{S}}\right] .
$$

Germano et al. ${ }^{29}$ contracted this tensor with the test-filtered rate-of-strain tensor $\bar{S}$ to obtain a scalar expression for $C_{\mathrm{dyn}}$,

$$
C_{\mathrm{dyn}} \Delta^{2}=\frac{\langle\boldsymbol{L}: \overline{\boldsymbol{S}}\rangle}{\langle\boldsymbol{Q}: \overline{\boldsymbol{S}}\rangle},
$$

where averaging over homogeneous directions is added to stabilize the procedure. Equation (26) ensures that $\mathbf{M}$ and $\overline{\mathbf{m}}+\mathbf{L}$ yield the same turbulent dissipation $\varepsilon_{t}$ at the $\mathcal{H} * \mathcal{G}$ filter level (with $\left.\varepsilon_{t}=\langle-\mathbf{M}: \overline{\mathbf{S}}\rangle\right){ }^{29}$

As an alternative, Lilly ${ }^{32}$ proposed to minimize, in a least-squares sense, the error between $\mathbf{L}$ and $C_{\mathrm{dyn}} \Delta^{2} \mathbf{Q}$. However, for the Smagorinsky model, differences between this approach and Eq. (26) are minimal, and for the slightly modified model, we consider in the current study (where the ensemble of the magnitude of the strain tensor $\langle 2 S: S\rangle$ is considered in the model), the result is identical. Moreover, for subgrid-scale models that do not follow the eddyviscosity approach, it was demonstrated recently ${ }^{33}$ that Germano's condition on the subgrid dissipation is best imposed as a constraint to Lilly's least-squares optimization. Therefore, we will concentrate in the current study on Germano's original formulation of the dynamic procedure, i.e., Eq. (26).

The popularity of the dynamic procedure nowadays is mainly related to its versatility when applied to complex simulations in which equilibrium and isotropy of the subgrid scales are not always satisfied. Moreover, it is presumed that the procedure predicts the correct asymptotic coefficient under high Reynolds number equilibrium conditions. In the current study, we can verify this by comparing the dynamic selection of $C_{s}=C_{\text {dyn }}^{1 / 2}$ as a function of the resolution $N$ to the multiobjective optimal trajectory obtained from the database analysis in Sec. IV A. To this end, we run dynamic Smagorinsky simulations at the same 14 resolutions $(16 \leq N \leq 256)$ as used for the setup of the LES database (cf. Sec. II). In our simulations, fluctuations in time of $C_{\text {dyn }}$ are very small such that we use a time-averaged value for comparison with the static optimal constants.

In Fig. 5, the results are shown, and the convergence of the dynamic trajectory to the asymptotic Smagorinsky constant is appreciated for resolutions that are sufficiently high (i.e., $N>160, L / \Delta>16$ ). At lower simulation resolutions, the dynamic coefficient deviates from the optimal trajectory. This occurs for $L / \bar{\Delta}<8$ (with $\bar{\Delta}$ being the width of the test filter). Hence, the Smagorinsky coefficient at test-filter level starts to depend on the marginal resolution of the integral length scale. This effect can be attenuated by using a scaledependent version of the dynamic procedure, as introduced by Porté-Agel et al. ${ }^{34}$ However, in the current work, the focus is on the asymptotic level (for $N \gg 1$ ) of the dynamic coefficient and how the effect of discretization and test-filter shape can be incorporated to preserve the correct asymptote.

First of all, it is instructive to use the dynamic procedure with discretization errors or with test-filter-shape inconsistencies in its standard form, i.e., using Eqs. (23), (25), and 


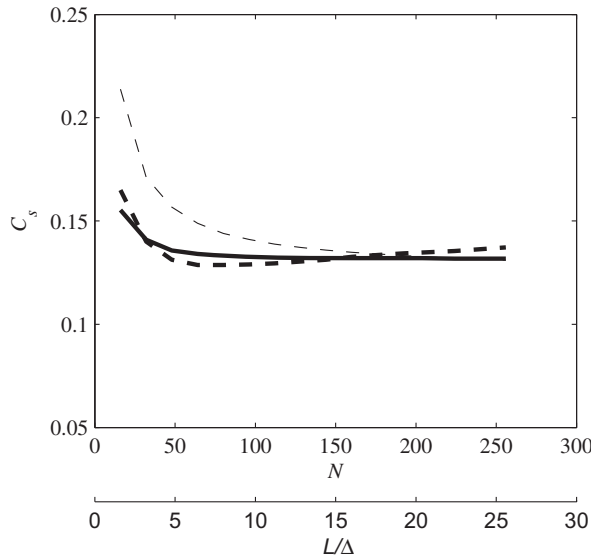

FIG. 5. Comparison of dynamic Smagorinsky trajectories with optimal trajectories. Pseudospectral discretization is employed, and for the dynamic procedure, a scale-similar test filter is used. (Bold line) Multiobjective optimal refinement trajectory based on $\widetilde{d}$ [cf. Eqs. (12) and (10)], (bold dashed) multiobjective optimal refinement trajectory based on $\widetilde{D}$ [cf. Eqs. (11) and (10)], and (- ) dynamic procedure using a scale-similar test filter.

(26). The effect of discretization on the dynamic trajectory is shown in Fig. 6(a). Here, it is clear that the use of a dynamic procedure without correction for discretization errors yields a dynamic coefficient, which is too low when compared to the multiobjective optimal strategy (for $N \gg 1$ ). In Fig. 6(b), the dynamic procedure is implemented using a top-hat test filter (discretized using the trapezoidal integration rule), which is not scale-similar to the cubical sharp-cut-off LES filter. Now the dynamic trajectory yields a coefficient that is considerably higher than the multiobjective optimal coefficient. These results suggest that there is considerable room for improvement of the dynamic procedure if the effects of discretization and filter-shape inconsistency can be accounted for. This is discussed next.

In a practical implementation of the dynamic procedure with discretization errors and filter inconsistencies, several elements affect the determination of the dynamic coefficient.
First, the filtered shear $S$ is now represented approximately, and we use the symbol $S_{d}$ to denote this. Second, the discrete test filter may not be scale-similar to the LES filter. We use the filter $\mathcal{F}$, with Fourier-kernel $F(\boldsymbol{k})$, to denote a test filter that is not scale-similar to the LES filter and further employ the notation $\mathcal{F} \tilde{\boldsymbol{u}}=\hat{\tilde{\boldsymbol{u}}}$. Taking all effects into account, the "discretized" dynamic procedure is then built using the following elements:

$$
\boldsymbol{L}_{\boldsymbol{d}}=\widehat{\widetilde{\boldsymbol{u}} \otimes \tilde{\boldsymbol{u}}}-\hat{\tilde{\boldsymbol{u}}} \otimes \hat{\tilde{u}}\left(\equiv \boldsymbol{T}_{\boldsymbol{d}}-\hat{\boldsymbol{\tau}}\right),
$$

and two terms $\boldsymbol{Q}_{\boldsymbol{d} 1}$ and $\boldsymbol{Q}_{\boldsymbol{d} 1}$ from the discretized representation of $\boldsymbol{Q}$, i.e.,

$$
\begin{aligned}
& \boldsymbol{Q}_{d 1}=-2 n\left\langle 2 \hat{S}_{d}: \hat{S}_{d}\right\rangle^{1 / 2} \hat{S}_{d}, \\
& \boldsymbol{Q}_{d 2}=\mathcal{F}\left[2\left\langle 2 \boldsymbol{S}_{d}: \boldsymbol{S}_{d}\right\rangle^{1 / 2} \boldsymbol{S}_{d}\right]=2\left\langle 2 \boldsymbol{S}_{d}: \boldsymbol{S}_{d}\right\rangle^{1 / 2} \hat{\boldsymbol{S}}_{\boldsymbol{d}} .
\end{aligned}
$$

Obviously combining the contractions $\left\langle\boldsymbol{L}_{d}: \hat{S}_{d}\right\rangle,\left\langle\boldsymbol{Q}_{d 1}: \hat{S}_{d}\right\rangle$, and $\left\langle\boldsymbol{Q}_{d 2}: \hat{S}_{d}\right\rangle$ straightforwardly in the dynamic procedure does not necessarily lead to the same result as their "exact" counterparts $\langle\boldsymbol{L}: \overline{\boldsymbol{S}}\rangle,\left\langle\boldsymbol{Q}_{1}: \overline{\boldsymbol{S}}\right\rangle$, and $\left\langle\boldsymbol{Q}_{2}: \overline{\boldsymbol{S}}\right\rangle$. Further, the level of the asymptote of the multiobjective optimal trajectory for $N \gg 1$ also depends on discretization errors, as discussed in Sec. IV A.

To reach proper high-Reynolds asymptotic behavior, we presume that the LES method is sufficiently reliable to yield a $k^{-5 / 3}$ spectrum when the correct model coefficient is selected by the dynamic procedure. It then becomes possible to estimate the different effects perturbing the dynamic procedure. We have the pseudospectral dynamic Smagorinsky results as a reference, for which it is appreciated that the dynamic procedure selects the correct asymptotic Smagorinsky coefficient (i.e., cf. the results in Fig. 5). Hence, it is sufficient to calibrate the average effects of the different terms in the dynamic procedure (i.e., $\left\langle\boldsymbol{L}_{d}: \hat{S}_{d}\right\rangle$, etc.) such that they correspond to their pseudospectral counterparts. In addition, as was demonstrated in Sec. IV A, the level of the Smagor-
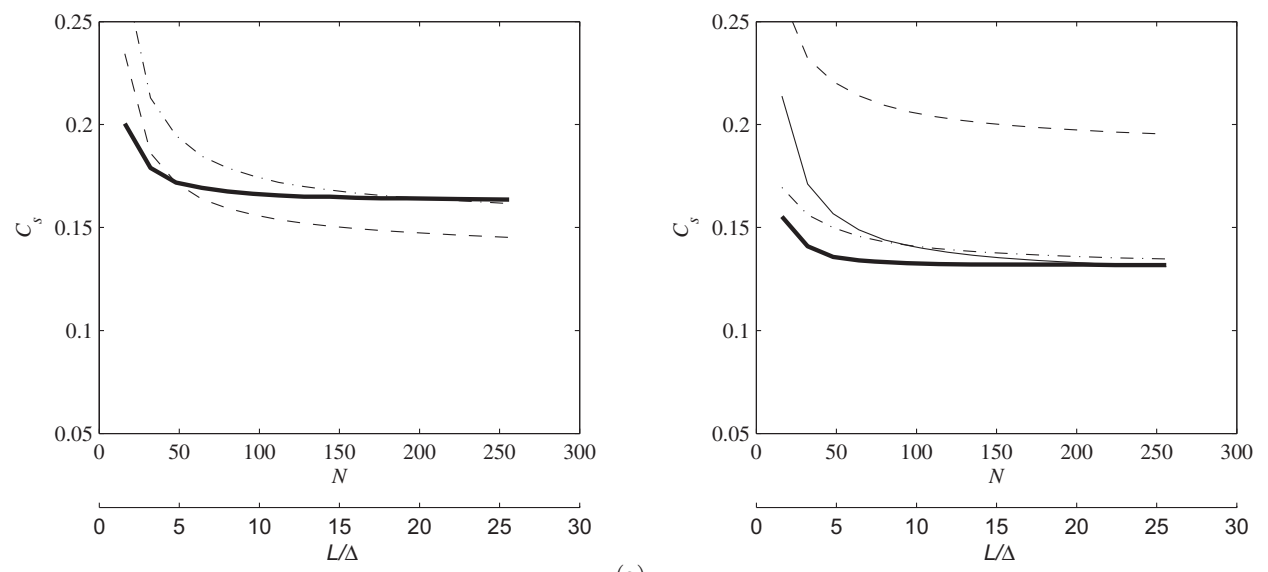

(a)

FIG. 6. Comparison of different dynamic trajectories with multiobjective trajectories. (a) The effect of discretization (using scale-similar test filters) in the presence of discretization errors. (Bold line) Multiobjective optimal refinement trajectory [cf. Eqs. (12) and (10)]: (- -) dynamic procedure without correction for discretization and (-.) dynamic procedure with correction for discretization. (b) The effect of scale-similarity of the test filter. (Bold line) Multiobjective optimal refinement trajectory [cf. Eqs. (12) and (10)]; (-) dynamic procedure using a scale-similar test filter; (- -) dynamic procedure using a top-hat test filter without correction; and (-.) dynamic procedure using a top-hat test filter with correction. 
TABLE I. Correction coefficients for the dynamic procedure, depending on different LES filters, test filters, and discretization schemes.

\begin{tabular}{|c|c|c|c|c|}
\hline \multirow[b]{2}{*}{ LES filter } & \multicolumn{4}{|c|}{ Discretization and test filter } \\
\hline & $\begin{array}{l}\text { Pseudospectral } \\
\text { scale-similar }\end{array}$ & $\begin{array}{l}\text { Pseudospectral } \\
\text { top-hat }^{\mathrm{a}}\end{array}$ & $\begin{array}{l}\text { Second-order } \\
\text { scale-similar }\end{array}$ & $\begin{array}{c}\text { Second-order } \\
\text { top-hat }^{\mathrm{a}}\end{array}$ \\
\hline \multirow{4}{*}{$\begin{array}{l}\text { Cubical sharp cut-off filter }(\gamma=1.22) \\
G(\boldsymbol{k})=\Pi_{i}\left[\mathbb{H}\left(k_{i}+k_{c}\right)-\mathbb{H}\left(k_{i}-k_{c}\right)\right]^{\mathrm{b}}\end{array}$} & $\gamma / \gamma_{d}=1.0$ & $\gamma / \gamma_{d}=1.0$ & $\gamma / \gamma_{d}=1.21$ & $\gamma / \gamma_{d}=1.21$ \\
\hline & $c_{1}=1.0$ & $c_{1}=0.931$ & $c_{1}=1.03$ & $c_{1}=0.956$ \\
\hline & $c_{2}=1.0$ & $c_{2}=2.34$ & $c_{2}=1.11$ & $c_{2}=2.54$ \\
\hline & $c_{3}=1.0$ & $c_{3}=1.76$ & $c_{3}=1.22$ & $c_{3}=2.12$ \\
\hline \multirow{4}{*}{$\begin{array}{l}\text { Top-hat } \star \text { cubical sco }(\gamma=0.870) \\
G(\boldsymbol{k})=\prod_{i} \sin \left(k_{i} \Delta / 2\right) /\left(k_{i} \Delta / 2\right) \times\left[\mathrm{H}\left(k_{i}+k_{c}\right)-\mathrm{H}\left(k_{i}-k_{c}\right)\right]^{\mathrm{b}}\end{array}$} & $\gamma / \gamma_{d}=1.0$ & $\gamma / \gamma_{d}=1.0$ & $\gamma / \gamma_{d}=1.15$ & $\gamma / \gamma_{d}=1.15$ \\
\hline & $c_{1}=1.0$ & $c_{1}=0.976$ & $c_{1}=1.02$ & $c_{1}=1.00$ \\
\hline & $c_{2}=1.0$ & $c_{2}=1.36$ & $c_{2}=1.08$ & $c_{2}=1.46$ \\
\hline & $c_{3}=1.0$ & $c_{3}=1.23$ & $c_{3}=1.16$ & $c_{3}=1.41$ \\
\hline \multirow[t]{4}{*}{ Spherical sharp cut-off filter $(\gamma=1.0) G(\boldsymbol{k})=\mathbb{H}\left(\|\boldsymbol{k}\|-k_{c}\right)^{\mathrm{b}}$} & $\gamma / \gamma_{d}=1.0$ & $\gamma / \gamma_{d}=1.0$ & $\gamma / \gamma_{d}=1.15$ & $\gamma / \gamma_{d}=1.15$ \\
\hline & $c_{1}=1.0$ & $c_{1}=1.00$ & $c_{1}=1.03$ & $c_{1}=1.03$ \\
\hline & $c_{2}=1.0$ & $c_{2}=1.57$ & $c_{2}=1.08$ & $c_{2}=1.70$ \\
\hline & $c_{3}=1.0$ & $c_{3}=1.35$ & $c_{3}=1.15$ & $c_{3}=1.56$ \\
\hline
\end{tabular}

${ }^{\mathrm{a}}$ We take a top-hat filter discretized using a trapezoidal integration rule, with a transfer function $H(\boldsymbol{k})=\Pi\left[1+\cos \left(k_{i} \Delta\right)\right] / 2$.

${ }^{\mathrm{b}} \mathrm{H}(k)$ is the Heaviside step function.

insky coefficient $C_{s}$ in the presence of discretization errors should be a factor $\gamma / \gamma_{d}$ higher than the coefficient of a pseudospectral discretization, and this may also easily be added into a "corrected" dynamic procedure. Therefore, we propose to introduce three correction factors into Eq. (26), i.e.,

$$
\begin{aligned}
C_{\mathrm{dyn}} \Delta^{2} & =\left(\frac{\gamma}{\gamma_{d}}\right)^{2} \frac{\langle\boldsymbol{L}: \overline{\boldsymbol{S}}\rangle}{\left\langle\boldsymbol{Q}_{1}: \overline{\boldsymbol{S}}\right\rangle+\left\langle\boldsymbol{Q}_{2}: \overline{\boldsymbol{S}}\right\rangle} \\
& =\left(\frac{\gamma}{\gamma_{d}}\right)^{2} \frac{c_{1}\left\langle\boldsymbol{L}_{\boldsymbol{d}}: \hat{\boldsymbol{S}}_{\boldsymbol{d}}\right\rangle}{c_{2}\left\langle\boldsymbol{Q}_{\boldsymbol{d} 1}: \hat{\boldsymbol{S}}_{\boldsymbol{d}}\right\rangle+c_{3}\left\langle\boldsymbol{Q}_{\boldsymbol{d} 2}: \hat{\boldsymbol{S}}_{\boldsymbol{d}}\right\rangle},
\end{aligned}
$$

with

$$
\begin{aligned}
& c_{1}=\langle\boldsymbol{L}: \overline{\boldsymbol{S}}\rangle /\left\langle\boldsymbol{L}_{d}: \hat{S}_{d}\right\rangle, \quad c_{2}=\left\langle\boldsymbol{Q}_{1}: \overline{\boldsymbol{S}}\right\rangle /\left\langle\boldsymbol{Q}_{\boldsymbol{d} 1}: \hat{\boldsymbol{S}}_{\boldsymbol{d}}\right\rangle, \quad \text { and } \\
& c_{3}=\left\langle\boldsymbol{Q}_{2}: \overline{\boldsymbol{S}}\right\rangle /\left\langle\boldsymbol{Q}_{\boldsymbol{d} 2}: \hat{\boldsymbol{S}}_{\boldsymbol{d}}\right\rangle .
\end{aligned}
$$

For high-Reynolds-number $k^{-5 / 3}$ spectra, $c_{1}, c_{2}$, and $c_{3}$ are constants, which can be expressed analytically similar to, e.g., the equations for $\gamma$ or $\gamma_{d}$. Detailed elaboration of this is included in Appendix B.

In Fig. 6, the results of a corrected dynamic procedure are also displayed next to "uncorrected" results that were already discussed above. To this end, Eq. (30) is used, and the values for $c_{1}, c_{2}$, and $c_{3}$ (shown in Table I) are obtained by numerically integrating Eqs. (B18), (B2), and (B5). Figure 6(a) shows that the effects of second-order discretization are now properly accounted for such that the dynamic coefficients at high simulation resolution correspond well to the multiobjective optimal trajectory. Also, the effect of a test filter that is not scale-similar to the LES filter can be properly accounted for, and this is demonstrated in Fig. 6(b) using a top-hat filter for the test filter.

\section{CONCLUSIONS}

A set of large-eddy simulations of high-Reynolds number decaying homogeneous isotropic turbulence was performed for systematic comparison with the active-grid turbulence of Kang et al. ${ }^{6}$ Comparing the LES results and experimental reference data, an error-landscape assessment was presented, following the error-landscape procedure developed in earlier work for DNS data. ${ }^{3,4}$ The use of experimental reference data allowed the evaluation of the optimal Smagorinsky coefficient at sufficiently high Reynolds numbers for a comparison to asymptotic analytical predictions of the Smagorinsky coefficient in the tradition of Lilly. ${ }^{9}$

Error-landscapes were constructed for a pseudospectral discretization and for an alternative discretization relying on the use of modified wavenumbers in the same spectral code. A robust characterization of multiobjective optimal trajectories was thus obtained, independent of the error measure $\left(d_{p}\right.$ or $D_{p}$ ), even when experimental reference data are used. The latter typically has less data points available compared to DNS data and may, of course, also exhibit uncertainties and experimental errors. It was found that near-optimal regions based on the integral length scale $\left(D_{-1}\right)$ did not overlap with other near-optimal regions. An argument was put forward that this deviation is related to the differences that exist between boundary conditions in the simulations and the experiments. In the latter, the walls of the wind tunnel dictated a flow domain significantly smaller than the computational box used in the simulations. Other flow properties (such as turbulent kinetic energy and enstrophy) were predicted quite well near the multiobjective optimal trajectories, and it was further demonstrated for multiobjective Smagorinsky coefficients that the LES spectra match the experimental $k^{-5 / 3}$ spectra very well at high wavenumbers.

The error-landscape methodology, applied at high Reynolds numbers, showed that the resulting multiobjective optimal trajectories have a horizontal asymptote at high simu- 
lation resolutions. For the pseudospectral results, this asymptotic optimal Smagorinsky coefficient was found to match very well Lilly's analytical predictions of the Smagorinsky coefficient ${ }^{9}$ when the shape of the LES filter (cubic spectral cutoff) is correctly taken into account. ${ }^{8}$ Also, the second-order multiobjective optimal trajectories showed a horizontal asymptote at high simulation resolutions, but now with a value of the coefficient that is a factor 1.21 higher than the pseudospectral coefficient. By slightly adapting Lilly's analysis to include effects of discretization, we were able to show that the optimal asymptotic second-order Smagorinsky coefficient also corresponds very well to the analytical predictions.

We further investigated the dynamic Smagorinsky model and compared the model coefficient in dynamic Smagorinsky LES to the multiobjective optimal trajectory obtained from the error-landscape analysis, similar to work in Refs. 4 and 20 at lower Reynolds numbers. For the pseudospectral discretization and employing a scale-similar test filter, it was found that the dynamic procedure converges well to the multiobjective optimal strategy and Lilly's analytical prediction of the model coefficient. However, in the presence of secondorder discretization errors, or test filters that are not scalesimilar to the LES filter (as may often occur in practical dynamic-model implementations), we found that the dynamic trajectory deviates considerably from the multiobjective trajectory. Based on the same analytical tools as used in Lilly's analysis of the standard Smagorinsky model, we elaborated a set of analytical correction factors, which account for discretization and test-filter-shape effects. When the dynamic procedure is properly corrected using these analytical correction factors, the dynamic trajectory converges to the expected Lilly asymptote at high simulation resolutions.

The high-Reynolds number asymptotic results in the current work are in our opinion important for the assessment of LES quality and reliability. They re-establish Lilly's analysis as a valuable tool for the calibration of model coefficients even in the presence of discretization errors. This presumes that the multiobjective optimal trajectory of the LES corresponds well to a $k^{-5 / 3}$ spectrum at high simulation resolutions and Reynolds numbers. We believe that this is a minimum requirement for the quality and reliability of a LES method, and the asymptotic convergence of its multiobjective optimal trajectory (for $\operatorname{Re} \gg 1$ and $N \gg 1$ ) to the "Lilly value" is a useful verification element for any LES method.

In the current work "optimal" trajectories obtained from an a posteriori analysis were used for the evaluation of the Smagorinsky model in high-Reynolds-number turbulence. Coincidentally, the somewhat similar term "optimal LES" is also used in the context of a priori testing, ${ }^{35}$ providing a theoretical framework for comparison of subgrid-scale models and the testing of new model formulations, e.g., using the concept of optimal estimators. ${ }^{36}$ The comparison of both methodologies and possible combination in a more global framework may be interesting topics for further research.

\section{APPENDIX A: ELABORATION OF $\langle S: S\rangle$ $\operatorname{AND}\left\langle S_{d}: S_{d}\right\rangle$}

An expression for $\langle\boldsymbol{S}: \boldsymbol{S}\rangle$ [i.e., Eq. (14) in Sec. IV A] is elaborated for homogeneous isotropic turbulence with a $k^{-5 / 3}$ spectrum. This relation is further used in Sec. IV A for the elaboration of $\gamma$ and the Smagorinsky coefficient $\left(C_{s}=C_{s, \infty} / \gamma\right)$. In order to elaborate the effect of discretization on the Smagorinsky constant in Sec. IV A, also an expression for $\left\langle S_{d}: S_{d}\right\rangle$ is required, where $S_{d}$ is the discretized representation of $S$. Presuming homogeneous isotropic turbulence and a $k^{-5 / 3}$ spectrum such an expression is also elaborated in the current appendix.

First of all, in continuous formulation

$$
\langle S: S\rangle=\langle\nabla \tilde{\boldsymbol{u}}: \nabla \tilde{\boldsymbol{u}}\rangle / 2+\left\langle\nabla \tilde{\boldsymbol{u}}:(\nabla \tilde{\boldsymbol{u}})^{T}\right\rangle / 2 .
$$

The second term on the right-hand side of Eq. (A1) disappears, i.e., using the continuity equation $\nabla \cdot \widetilde{\boldsymbol{u}}=0$, it is readily shown that

$$
\left\langle\nabla \tilde{\boldsymbol{u}}:(\nabla \tilde{\boldsymbol{u}})^{T}\right\rangle=\langle\nabla \cdot[\nabla \cdot(\tilde{\boldsymbol{u}} \otimes \tilde{\boldsymbol{u}})]\rangle=\nabla \cdot\langle\nabla \cdot(\tilde{\boldsymbol{u}} \otimes \tilde{\boldsymbol{u}})\rangle=0,
$$

and the divergence of an ensemble average of a vector field in homogeneous turbulence [cf. right-hand side of Eq. (A2)] is readily shown to be zero.

For homogeneous turbulence, the term $\langle\nabla \tilde{\boldsymbol{u}}: \nabla \tilde{\boldsymbol{u}}\rangle$ may now be represented as an integral in Fourier space, i.e.,

$$
\langle\nabla \tilde{\boldsymbol{u}}: \nabla \tilde{\boldsymbol{u}}\rangle=\iiint\langle\{\nabla \tilde{\boldsymbol{u}}\}(\boldsymbol{k}):\{\nabla \tilde{\boldsymbol{u}}\}(-\boldsymbol{k})\rangle \mathrm{d} \boldsymbol{k},
$$

with $\{\nabla \tilde{\boldsymbol{u}}\}(\boldsymbol{k})$ being the Fourier transform of $\nabla \tilde{\boldsymbol{u}}$. The above identity is nontrivial as it involves stochastic integrals expressed as an integral over space in the limit of a spatial integration domain going to infinity. A rigorous mathematical treatment of this can be found in Ref. 37; a more easy-tograsp work-around is, e.g., presented in Ref. 16.

We now use (with $\{\tilde{\boldsymbol{u}}\}$ being the Fourier transform of $\tilde{\boldsymbol{u}}$ )

$$
\{\nabla \tilde{\boldsymbol{u}}\}(\boldsymbol{k})=\imath \boldsymbol{k} \otimes\{\tilde{\boldsymbol{u}}\}(\boldsymbol{k})=\imath \boldsymbol{k} \otimes\{\boldsymbol{u}\}(\boldsymbol{k}) G(\boldsymbol{k})
$$

to elaborate

$$
\langle\boldsymbol{S}: \boldsymbol{S}\rangle=\frac{1}{2} \iiint k^{2}\langle\{\boldsymbol{u}\}(\boldsymbol{k}) \cdot\{\boldsymbol{u}\}(-\boldsymbol{k})\rangle[G(\boldsymbol{k})]^{2} \mathrm{~d} \boldsymbol{k} .
$$

Finally, for isotropic turbulence, the relation $\langle\{\boldsymbol{u}\}(\boldsymbol{k}) \cdot\{\boldsymbol{u}\}(-\boldsymbol{k})\rangle=E(k) /\left(2 \pi k^{2}\right) \quad$ (cf., e.g., Ref. 16) is employed. Further presuming $E(k)=C_{K} \varepsilon^{2 / 3} k^{-5 / 3}$ leads to

$$
\begin{aligned}
\langle\boldsymbol{S}: \boldsymbol{S}\rangle & =\frac{1}{4 \pi} \iiint E(k)[G(\boldsymbol{k})]^{2} \mathrm{~d} \boldsymbol{k} \\
& =\frac{1}{4 \pi} \iiint C_{K} \varepsilon^{2 / 3} k^{-5 / 3}[G(\boldsymbol{k})]^{2} \mathrm{~d} \boldsymbol{k} .
\end{aligned}
$$

Now $\left\langle S_{d}: S_{d}\right\rangle$ is further elaborated. In a discretized formulation, Eq. (A2) is not guaranteed: in particular, $\left\langle\nabla_{\boldsymbol{d}} \tilde{\boldsymbol{u}}:\left(\nabla_{\boldsymbol{d}} \tilde{\boldsymbol{u}}\right)^{T}\right\rangle \neq\left\langle\nabla_{\boldsymbol{d}} \cdot\left[\nabla_{\boldsymbol{d}} \cdot(\tilde{\boldsymbol{u}} \otimes \tilde{\boldsymbol{u}})\right]\right\rangle$. The equality in the discretized version depends on additional symmetry properties of the selected discrete derivative operators. ${ }^{38}$ Here, we will 
presume that the difference is small (on the order of the discretization error) such that we can approximate

$$
\left\langle S_{d}: S_{d}\right\rangle \approx\left\langle\nabla_{d} \tilde{u}: \nabla_{d} \tilde{u}\right\rangle / 2 .
$$

Further elaboration is now similar to the continuous case, but with the difference that we use modified wavenumbers to elaborate $\left\{\nabla_{\boldsymbol{d}} \tilde{\boldsymbol{u}}\right\}(\boldsymbol{k})$ (i.e., the Fourier transform of $\nabla_{\boldsymbol{d}} \widetilde{\boldsymbol{u}}$ ). Using the modified wavenumbers for first derivatives as used in the second-order discretization of the current work [cf. Eq. (6)], we find

$$
\left\{\nabla_{\boldsymbol{d}} \tilde{\boldsymbol{u}}\right\}(\boldsymbol{k})=l \frac{\sin (\boldsymbol{k} \Delta / 2)}{\Delta / 2} \otimes\{\tilde{\boldsymbol{u}}\}(\boldsymbol{k}) .
$$

This leads to

$$
\begin{aligned}
\left\langle\boldsymbol{S}_{\boldsymbol{d}}: \boldsymbol{S}_{\boldsymbol{d}}\right\rangle & =\frac{1}{2} \iiint \sum_{i=1}^{3}\left[\frac{\sin \left(k_{i} \Delta / 2\right)}{\Delta / 2}\right]^{2}\langle\{\tilde{\boldsymbol{u}}\}(\boldsymbol{k}) \cdot\{\tilde{\boldsymbol{u}}\}(-\boldsymbol{k})\rangle \mathrm{d} \boldsymbol{k} \\
& =\frac{1}{4 \pi} \iiint \sum_{i=1}^{3}\left[\frac{\sin \left(k_{i} \Delta / 2\right)}{k \Delta / 2}\right]^{2} E(k)[G(\boldsymbol{k})]^{2} \mathrm{~d} \boldsymbol{k} .
\end{aligned}
$$

Further elaboration, presuming $E(k)=C_{K} \varepsilon^{2 / 3} k^{-5 / 3}$, leads to

$$
\left\langle S_{d}: S_{d}\right\rangle \approx \frac{1}{4 \pi} \iiint C_{K} \varepsilon^{2 / 3} k^{-5 / 3}[G(k)]^{2} G_{D}(\boldsymbol{k}) \mathrm{d} \boldsymbol{k},
$$

with

$$
G_{D}(\boldsymbol{k})=\sum_{i=1}^{3}\left[\frac{\sin \left(k_{i} \Delta / 2\right)}{k \Delta / 2}\right]^{2}=2 \frac{\sum_{i=1}^{3} 1-\cos \left(k_{i} \Delta\right)}{(k \Delta)^{2}} .
$$

\section{APPENDIX B: CORRECTIONS TO THE DYNAMIC PROCEDURE AT HIGH REYNOLDS NUMBERS}

In this appendix, the constants $c_{1}, c_{2}$, and $c_{3}$ are elaborated for high-Reynolds-number $k^{-5 / 3}$ spectra. The determination of $c_{2}$ and $c_{3}$ is straightforward. It relies on the relation [cf. Eqs. (14)-(17)]

$$
\langle 2 S: S\rangle^{3 / 2}=\left\{2 \int_{0}^{\infty} C_{K} \varepsilon^{2 / 3} k^{1 / 3}[G(\boldsymbol{k})]^{2} \mathrm{~d} k\right\}^{3 / 2}=\varepsilon\left(\frac{\gamma}{C_{s, \infty} \Delta}\right)^{2}
$$

and similar relations for $\left\langle 2 \hat{S}_{d}: \hat{S}_{d}\right\rangle$, etc. Hence,

$$
c_{2}=\frac{\left\langle Q_{1}: \bar{S}\right\rangle}{\left\langle Q_{d 1}: \hat{S}_{d}\right\rangle}=\frac{\langle 2 \bar{S}: \bar{S}\rangle^{3 / 2}}{\left\langle 2 \hat{S}_{d}: \hat{S}_{d}\right\rangle^{3 / 2}}=\left(\frac{\bar{\gamma}}{\hat{\gamma}_{d}}\right)^{2}
$$

with [comparable to Eqs. (17) and (18)]

$$
\bar{\gamma}=\frac{\left(\frac{1}{3 \pi} \iiint_{-\infty}^{\infty} k^{-5 / 3}[H(\boldsymbol{k}) G(\boldsymbol{k})]^{2} \mathrm{~d} \boldsymbol{k}\right)^{3 / 4}}{\pi / \Delta},
$$

$$
\hat{\gamma}_{d}=\frac{\left(\frac{1}{3 \pi} \iiint_{-\infty}^{\infty} k^{-5 / 3}[F(\boldsymbol{k}) G(\boldsymbol{k})]^{2} G_{D}(\boldsymbol{k}) \mathrm{d} \boldsymbol{k}\right)^{3 / 4}}{\pi / \Delta} .
$$

As may be expected, in the absence of discretization errors and for a scale-similar test filter [such that $H(\boldsymbol{k})=F(\boldsymbol{k})$ ], $\bar{\gamma}=\hat{\gamma}_{d}$ and $c_{2}=1$. Also, using Eq. (22), it is readily shown that $\bar{\gamma}=\gamma / n$.

For $c_{3}$, we have

$$
c_{3}=\frac{\left\langle\boldsymbol{Q}_{2}: \overline{\boldsymbol{S}}\right\rangle}{\left\langle\boldsymbol{Q}_{\boldsymbol{d} 2}: \hat{\boldsymbol{S}}_{\boldsymbol{d}}\right\rangle}=\frac{\langle 2 \boldsymbol{S}: \boldsymbol{S}\rangle^{1 / 2}\langle 2 \overline{\boldsymbol{S}}: \overline{\boldsymbol{S}}\rangle}{\left\langle 2 \boldsymbol{S}_{\boldsymbol{d}}: \boldsymbol{S}_{\boldsymbol{d}}\right\rangle^{1 / 2}\left\langle 2 \hat{\boldsymbol{S}}_{\boldsymbol{d}}: \hat{\boldsymbol{S}}_{\boldsymbol{d}}\right\rangle}=\left(\frac{\gamma}{\gamma_{d}}\right)^{2 / 3}\left(\frac{\bar{\gamma}}{\hat{\gamma}_{d}}\right)^{4 / 3} .
$$

In the absence of discretization errors and for a scale-similar test filter, it is clear that $c_{3}=1$. The fact that both $\gamma_{d}$ and $\hat{\gamma}_{d}$ appear in the expression for $c_{3}$ reflects that discretization errors typically affect the LES field differently at LES filter level than at test filter level.

The analysis of $\langle\boldsymbol{L}: \overline{\boldsymbol{S}}\rangle$ and $\left\langle\boldsymbol{L}_{\boldsymbol{d}}: \hat{\boldsymbol{S}}_{\boldsymbol{d}}\right\rangle$ for the determination of $c_{1}$ is more difficult since $\boldsymbol{L}$ and $\boldsymbol{L}_{\boldsymbol{d}}$ represent nonlinear tensors. We first focus on $\langle\boldsymbol{L}: \overline{\boldsymbol{S}}\rangle$. Since we are considering high Reynolds numbers and high simulation resolutions such that both LES and test filter are situated in a $k^{-5 / 3}$ inertial range, we can presume a constant energy flux at both filter levels. Hence, $\langle\mathbf{T}: \overline{\boldsymbol{S}}\rangle=-\varepsilon$ and $\langle\boldsymbol{\tau}: \boldsymbol{S}\rangle=-\varepsilon$, with $\varepsilon$ being the total dissipation. Using this, we express

$$
\begin{aligned}
\langle\mathbf{L}: \overline{\boldsymbol{S}}\rangle & =\langle\mathbf{T}: \overline{\boldsymbol{S}}\rangle-\langle\overline{\boldsymbol{\tau}}: \overline{\boldsymbol{S}}\rangle \\
& =-\varepsilon-\langle\overline{\boldsymbol{\tau}}: \overline{\boldsymbol{S}}\rangle=-\varepsilon-\langle\boldsymbol{\tau}: \overline{\bar{S}}\rangle \\
& =\left\langle\boldsymbol{\tau}: \boldsymbol{S}^{\prime \prime}\right\rangle \\
& =\langle\boldsymbol{\tau}: \boldsymbol{\tau}\rangle^{1 / 2}\left\langle\boldsymbol{S}^{\prime \prime}: \boldsymbol{S}^{\prime \prime}\right\rangle^{1 / 2} \cos \bar{\alpha}
\end{aligned}
$$

with $S^{\prime \prime}=S-\overline{\bar{S}}$ and $\bar{\alpha}$ being the statistical angle between $\boldsymbol{\tau}$ and $S^{\prime \prime}$. The second equality in Eq. (B7) holds for self-adjoint filters, ${ }^{39}$ where the ensemble operator $\langle\cdot\rangle$ is used as the definition of the inner product. ${ }^{8}$ The convolution filters considered in the current paper are easily shown to be self-adjoint in this inner-product space. ${ }^{8}$

The term $\left\langle\boldsymbol{L}_{\boldsymbol{d}}: \hat{\boldsymbol{S}}_{\boldsymbol{d}}\right\rangle$ is further elaborated in two steps. First,

$$
\left\langle\boldsymbol{L}_{\boldsymbol{d}}: \hat{\boldsymbol{S}}_{\boldsymbol{d}}\right\rangle=\left\langle\boldsymbol{L}_{\boldsymbol{d}}: \boldsymbol{L}_{\boldsymbol{d}}\right\rangle^{1 / 2}\left\langle\hat{\boldsymbol{S}}_{\boldsymbol{d}}: \hat{\boldsymbol{S}}_{\boldsymbol{d}}\right\rangle^{1 / 2} \cos \hat{\beta}_{d}
$$

and

$$
\left\langle\boldsymbol{L}_{\boldsymbol{d}}: \hat{\boldsymbol{S}}\right\rangle=\left\langle\boldsymbol{L}_{\boldsymbol{d}}: \boldsymbol{L}_{\boldsymbol{d}}\right\rangle^{1 / 2}\langle\hat{\boldsymbol{S}}: \hat{\boldsymbol{S}}\rangle^{1 / 2} \cos \hat{\boldsymbol{\beta}},
$$

with $\hat{\beta}$ and $\hat{\beta}_{d}$ being the statistical angle between $\boldsymbol{L}_{\boldsymbol{d}}$ and $\hat{\boldsymbol{S}}$ and between $\boldsymbol{L}_{\boldsymbol{d}}$ and $\hat{S}_{\boldsymbol{d}}$, respectively. Hence, using Eqs. (B10) and (B11)

$$
\left\langle\boldsymbol{L}_{\boldsymbol{d}}: \hat{\boldsymbol{S}}_{\boldsymbol{d}}\right\rangle=\left\langle\boldsymbol{L}_{\boldsymbol{d}}: \hat{\boldsymbol{S}}\right\rangle\left(\frac{\hat{\gamma}_{d}}{\hat{\gamma}}\right)^{2 / 3} \frac{\cos \hat{\beta}_{d}}{\cos \hat{\beta}}
$$

where $\hat{\gamma}$ is defined similar to $\bar{\gamma}[\mathrm{Eq}$. (B3)]. 
Second, the term $\left\langle\boldsymbol{L}_{d}: \hat{\boldsymbol{S}}\right\rangle=\left\langle\left(\boldsymbol{T}_{\boldsymbol{d}}-\hat{\boldsymbol{\tau}}\right): \hat{\boldsymbol{S}}\right\rangle$ can now be further elaborated. It is recognized that $\boldsymbol{T}_{\boldsymbol{d}}$ and $\hat{\boldsymbol{S}}$ are the subgrid stress and strain tensor at the $(\mathcal{F} * \mathcal{G})$-filter level, respectively. Thus, for a high-Reynolds-number inertial-range spectrum, we also have $\left\langle\boldsymbol{T}_{d}: \hat{\boldsymbol{S}}\right\rangle=-\varepsilon$. Consequently [similar to Eq. (B9)],

$$
\begin{aligned}
\left\langle\boldsymbol{L}_{d}: \hat{\boldsymbol{S}}\right\rangle & =\left\langle\boldsymbol{T}_{d}: \hat{\boldsymbol{S}}\right\rangle-\langle\hat{\boldsymbol{\tau}}: \hat{\boldsymbol{S}}\rangle=\varepsilon-\langle\boldsymbol{\tau}: \hat{\hat{\boldsymbol{S}}}\rangle=\left\langle\boldsymbol{\tau}: \boldsymbol{S}^{* * *}\right\rangle \\
& =\langle\boldsymbol{\tau}: \boldsymbol{\tau}\rangle^{1 / 2}\left\langle\boldsymbol{S}^{* *}: \boldsymbol{S}^{* *}\right\rangle^{1 / 2} \cos \hat{\alpha},
\end{aligned}
$$

with $S^{* *}=S-\hat{\hat{S}}$ and $\hat{\alpha}$ being the angle between $\boldsymbol{\tau}$ and $S^{* *}$.

By bringing Eqs. (B9), (B12), and (B14) together, we find

$$
\begin{aligned}
c_{1} & =\frac{\langle\boldsymbol{L}: \overline{\boldsymbol{S}}\rangle}{\left\langle\boldsymbol{L}_{\boldsymbol{d}}: \hat{S}_{\boldsymbol{d}}\right\rangle}=\frac{\left\langle\boldsymbol{S}^{\prime \prime}: \boldsymbol{S}^{\prime \prime}\right\rangle^{1 / 2} \cos \bar{\alpha}}{\left\langle\boldsymbol{S}^{* *}: \boldsymbol{S}^{* * *}\right\rangle^{1 / 2} \cos \hat{\alpha}}\left(\frac{\hat{\gamma}}{\hat{\gamma}_{d}}\right)^{2 / 3} \frac{\cos \hat{\beta}}{\cos \hat{\beta}_{d}} \\
& =\left(\frac{\gamma^{\prime}}{\gamma^{* *}}\right)^{2 / 3}\left(\frac{\hat{\gamma}}{\hat{\gamma}_{d}}\right)^{2 / 3} \frac{\cos \bar{\alpha}}{\cos \hat{\alpha}} \frac{\cos \hat{\beta}}{\cos \hat{\beta}_{d}},
\end{aligned}
$$

with

$$
\gamma^{\prime}=\frac{\left(\frac{1}{3 \pi} \iiint_{-\infty}^{\infty} k^{-5 / 3}\left[G(\boldsymbol{k})-H(\boldsymbol{k})^{2} G(\boldsymbol{k})\right]^{2} \mathrm{~d} \boldsymbol{k}\right)^{3 / 4}}{\pi / \Delta},
$$

$$
\gamma^{* *}=\frac{\left(\frac{1}{3 \pi} \iiint_{-\infty}^{\infty} k^{-5 / 3}\left[G(\boldsymbol{k})-F(\boldsymbol{k})^{2} G(\boldsymbol{k})\right]^{2} \mathrm{~d} \boldsymbol{k}\right)^{3 / 4}}{\pi / \Delta} .
$$

The main unknowns remaining in Eq. (B15) are $\bar{\alpha}, \hat{\alpha}, \hat{\beta}$, and $\hat{\beta}_{d}$. In the present paper, we make the strong assumption that $\cos \bar{\alpha} / \cos \hat{\alpha} \approx 1$ and $\cos \hat{\beta} / \cos \hat{\beta}_{d} \approx 1$ such that

$$
c_{1} \approx\left(\frac{\gamma^{\prime \prime}}{\gamma^{* *}}\right)^{2 / 3}\left(\frac{\hat{\gamma}}{\hat{\gamma}_{d}}\right)^{2 / 3}
$$

As demonstrated in Sec. IV B, this leads to very satisfactory corrections to the dynamic procedure.

Equations (B2), (B5), and (B18) can now be calculated by numerical integration of Eqs. (17), (18), (B3), (B4), (B16), and (B17). In Table I, an overview of the coefficients is presented for different LES filter and test-filter shapes and for either a pseudospectral or second-order discretization. As appreciated from this table, the corrections may differ significantly from unity when discretization, and test-filter effects play a role.

${ }^{1}$ P. Gualtieri, C. M. Casciola, R. Benzi, and R. Piva, "Preservation of statistical properties in large-eddy simulation of shear turbulence," J. Fluid Mech. 592, 471 (2007).

${ }^{2}$ N. Park and K. Mahesh, "Analysis of numerical errors in large eddy simulation using statistical closure theory," J. Comput. Phys. 222, 194 (2007). ${ }^{3}$ J. Meyers, B. J. Geurts, and P. Sagaut, "A computational error assessment of central finite-volume discretizations in large-eddy simulation using a Smagorinsky model,” J. Comput. Phys. 227, 156 (2007).
${ }^{4}$ J. Meyers, P. Sagaut, and B. J. Geurts, "Optimal model parameters for multi-objective large-eddy simulations," Phys. Fluids 18, 095103 (2006).

${ }^{5}$ J. Meyers, B. J. Geurts, and M. Baelmans, "Database-analysis of errors in large-eddy simulation," Phys. Fluids 15, 2740 (2003).

${ }^{6}$ H. S. Kang, S. Chester, and C. Meneveau, "Decaying turbulence in an active-grid-generated flow and comparisons with large-eddy simulation," J. Fluid Mech. 480, 129 (2003).

${ }^{7}$ J. Meyers and M. Baelmans, "Determination of subfilter energy in largeeddy simulations," J. Turbul. 5, 26 (2004).

${ }^{8}$ J. Meyers and P. Sagaut, "On the model coefficients for the standard and the variational multi-scale Smagorinsky model," J. Fluid Mech. 569, 287 (2006).

${ }^{9}$ D. K. Lilly, "The representation of small-scale turbulence in numerical simulation experiments," Proceedings of IBM Scientific Computing Symposium on Environmental Sciences, IBM Data Processing Division, White Plains, New York, 1967.

${ }^{10}$ N. N. Mansour, P. Moin, W. C. Reynolds, and J. H. Ferziger, in Turbulent Shear Flows I, edited by F. Durst, B. E. Launder, F. W. Schmidt, and J. H. Whitelaw (Springer, Berlin, 1979), pp. 286-401.

${ }^{11} \mathrm{~S}$. Ghosal, "An analysis of numerical errors in large-eddy simulations of turbulence," J. Comput. Phys. 125, 187 (1996).

${ }^{12}$ B. Vreman, B. Geurts, and H. Kuerten, "Comparison of numerical schemes in large-eddy simulations of the temporal mixing layer," Int. J. Numer. Methods Fluids 22, 297 (1996).

${ }^{13}$ B. J. Geurts and J. Fröhlich, "A framework for predicting accuracy limitations in large eddy simulations," Phys. Fluids 14, L41 (2002).

${ }^{14}$ I. Fedioun, N. Lardjane, and I. Gökalp, "Revisiting numerical errors in direct and large eddy simulations of turbulence: Physical and spectral analysis," J. Comput. Phys. 174, 816 (2001).

${ }^{15} \mathrm{~A}$. Muschinski, "A similarity theory of locally homogeneous and isotropic turbulence generated by a Smagorinsky-type LES," J. Fluid Mech. 325, 239 (1996).

${ }^{16}$ S. B. Pope, Turbulent Flows (Cambridge University Press, Cambridge, 2000).

${ }^{17}$ J.-C. Magnient, P. Sagaut, and M. Deville, "A study of built-in filter for some eddy viscosity models in large-eddy simulation," Phys. Fluids 13, 1440 (2001).

${ }^{18}$ R. Cocle, L. Bricteux, and G. Winckelmans, "Scale dependence and asymptotic very high Reynolds number spectral behavior of multiscale subgrid models," Phys. Fluids 21, 085101 (2009).

${ }^{19}$ C. Fureby and F. F. Grinstein, "Monotonically integrated large eddy simulation of free shear flows," AIAA J. 37, 544 (1999).

${ }^{20}$ J. Meyers, B. J. Geurts, and M. Baelmans, "Optimality of the dynamic procedure for large-eddy simulations," Phys. Fluids 17, 045108 (2005).

${ }^{21}$ J. Smagorinsky, "General circulation experiments with the primitive equations: I. The basic experiment," Mon. Weather Rev. 91, 99 (1963).

${ }^{22}$ O. J. McMillan and J. H. Ferziger, "Direct testing of subgrid-scale models," AIAA J. 17, 1340 (1979).

${ }^{23}$ S. Cerutti, C. Meneveau, and O. M. Knio, "Spectral and hyper eddy viscosity in high-Reynolds-number turbulence," J. Fluid Mech. 421, 307 (2000).

${ }^{24}$ J. Meyers and C. Meneveau, "A functional form for the energy spectrum parameterizing bottleneck and intermittency effects," Phys. Fluids 20, 065109 (2008).

${ }^{25}$ C. Canuto, M. Y. Hussaini, A. Quarteroni, and T. A. Zang, Spectral Methods in Fluid Dynamics, Springer Series in Computational Physics (Springer-Verlag, Berlin, Heidelberg, New York, 1988).

${ }^{26}$ J. H. Ferziger and M. Perić, Computational Methods for Fluid Dynamics (Springer-Verlag, Berlin, Heidelberg, New York, 1997).

${ }^{27} \mathrm{C}$. Meneveau, "Statistics of turbulence subgrid-scale stresses: Necessary conditions and experimental tests," Phys. Fluids 6, 815 (1994).

${ }^{28}$ B. J. Geurts and F. van der Bos, "Numerically induced high-pass dynamics in large-eddy simulation," Phys. Fluids 17, 125103 (2005).

${ }^{29}$ M. Germano, U. Piomelli, P. Moin, and W. H. Cabot, "A dynamic subgridscale eddy viscosity model," Phys. Fluids A 3, 1760 (1991).

${ }^{30}$ D. Carati and E. Vanden Eijnden, "On the self-similarity assumption in dynamic models for large eddy simulations," Phys. Fluids 9, 2165 (1997).

${ }^{31}$ M. Germano, "Turbulence: The filtering approach," J. Fluid Mech. 238, 325 (1992).

${ }^{32}$ D. K. Lilly, "A proposed modification of the Germano subgrid-scale closure method," Phys. Fluids A 4, 633 (1992).

${ }^{33}$ Y. Shi, Z. Xiao, and S. Chen, "Constrained subgrid-scale stress model for large eddy simulation," Phys. Fluids 20, 011701 (2008). 
${ }^{34}$ F. Porté-Agel, C. Meneveau, and M. B. Parlange, "A scale-dependent dynamic model for large-eddy simulation: Application to a neutral atmospheric boundary layer," J. Fluid Mech. 415, 261 (2000).

${ }^{35}$ J. A. Langford and R. D. Moser, "Optimal LES formulations for isotropic turbulence," J. Fluid Mech. 398, 321 (1999).

${ }^{36} \mathrm{G}$. Balarac, H. Pitsch, and V. Raman, "Development of a dynamic model for the subfilter scalar variance using the concept of optimal estimators," Phys. Fluids 20, 035114 (2008).

${ }^{37} \mathrm{G}$. K. Batchelor, The Theory of Homogeneous Turbulence, Cambridge Monographs on Mechanics and Applied Mathematics (Cambridge University Press, Cambridge, 1953).
${ }^{38}$ R. W. C. P. Verstappen and A. E. P. Veldman, "Symmetry-preserving discretization of turbulent flow," J. Comput. Phys. 187, 343 (2003).

${ }^{39} \mathrm{~A}$. W. Vreman, "The adjoint filter operator in large-eddy simulation of turbulent flow," Phys. Fluids 16, 2012 (2004).

${ }^{40}$ For clarity of notation in the derivations of Sec. IV and Appendixes A and B, the bar usually added on top of the symbol $S$ to denote the filtered rate-of-strain tensor is omitted. More conventional notations and an introduction to subgrid-scale modeling may be found in Ref. 41.

${ }^{41}$ C. Meneveau, "Turbulence: Subgrid-scale modeling," Scholarpedia J. 5, 9489 (2010). 